\title{
SUPERLUMINOUS SPIRAL GALAXIES
}

\author{
Patrick M. Ogle ${ }^{1}$, Lauranne Lanz ${ }^{1}$, Cyril Nader ${ }^{1,2}$, and George Helou ${ }^{1}$ \\ ${ }^{1}$ IPAC, California Institute of Technology, Mail Code 220-6, Pasadena, CA 91125, USA; ogle@ipac.caltech.edu \\ ${ }^{2}$ University of California, Los Angeles, California \\ Received 2015 October 30; accepted 2015 December 17; published 2016 January 26
}

\begin{abstract}
We report the discovery of spiral galaxies that are as optically luminous as elliptical brightest cluster galaxies, with $r$-band monochromatic luminosity $L_{r}=8-14 L^{*}\left(4.3-7.5 \times 10^{44} \mathrm{erg} \mathrm{s}^{-1}\right)$. These super spiral galaxies are also giant and massive, with diameter $D=57-134 \mathrm{kpc}$ and stellar mass $M_{\text {stars }}=0.3-3.4 \times 10^{11} M_{\odot}$. We find 53 super spirals out of a complete sample of 1616 SDSS galaxies with redshift $z<0.3$ and $L_{r}>8 L^{*}$. The closest example is found at $z=0.089$. We use existing photometry to estimate their stellar masses and star formation rates (SFRs). The SDSS and Wide-field Infrared Survey Explorer colors are consistent with normal star-forming spirals on the blue sequence. However, the extreme masses and rapid SFRs of 5-65 $M_{\odot} \mathrm{yr}^{-1}$ place super spirals in a sparsely populated region of parameter space, above the star-forming main sequence of disk galaxies. Super spirals occupy a diverse range of environments, from isolation to cluster centers. We find four super spiral galaxy systems that are late-stage major mergers-a possible clue to their formation. We suggest that super spirals are a remnant population of unquenched, massive disk galaxies. They may eventually become massive lenticular galaxies after they are cut off from their gas supply and their disks fade.
\end{abstract}

Key words: galaxies: spiral

\section{INTRODUCTION}

The most massive galaxies in the universe are thought to form from the largest density peaks in the primordial matter distribution. Galaxy mergers change the initial galaxy mass function, forming more massive galaxies by combining less massive ones. The result is the galaxy mass distribution we see in the local universe, empirically described by the Schechter (1976) luminosity function, together with a morphologydependent mass-to-light ratio. The luminosity function also depends upon the star formation history of galaxies, regulated by gas content, gas accretion, stellar feedback, and active galactic nucleus (AGN) feedback. Galaxy mergers play an important role here too, since tidal torques in merging systems force gas into the galaxy centers, leading to starburst activity that grows the stellar bulge and AGN activity that grows the supermassive black hole (Toomre \& Toomre 1972; Barnes \& Hernquist 1991; Hopkins et al. 2009).

Galaxies segregate into two major classes based on color and morphology (Strateva et al. 2001; Lintott et al. 2008). Blue, star-forming disks (late-type galaxies, LTGs) lie in one region of color-space called the blue-sequence. Red-and-dead spheroids (early-type galaxies, ETGs) lie in a different region of color space called the red sequence. LTGs demonstrate a correlation between star formation rate (SFR) and stellar mass $\left(M_{\text {stars }}\right)$ called the star-forming main sequence (SFMS: Brinchmann et al. 2004; Elbaz et al. 2007; Wuyts et al. 2011). The SFMS may be a consequence of an equilibrium between inflowing gas and star-formation driven outflows, with the specific star formation rate (SSFR) regulated by the halo mass growth rate (Lilly et al. 2013). Most ETGs on the other hand have much lower SFRs because they lack the cold gas needed to sustain star formation.

It appears that there is a limit to the mass of star-forming disk galaxies of roughly $3 \times 10^{10} M_{\odot}$, with the most massive disk galaxies transitioning away from the main sequence as their SSFR declines. This decline appears to be a gradual process, occurring over a period longer than $1 \mathrm{Gyr}$ after the gas supply to the galaxy disk has been interrupted (Schawinski et al. 2014). Rapid quenching does not appear to occur for most galaxies that remain disk galaxies, contrary to early attempts to explain the apparently bimodal distribution of galaxy colors.

A number of mechanisms have been suggested to explain why the gas supply is interrupted for the most massive disk galaxies. Major galaxy mergers may disrupt merging disk galaxies and transform them rapidly into elliptical galaxies (Baldry et al. 2004), though this does not explain the transformation of galaxies that remain disks. The accretion of cold gas onto a galaxy may be stopped when the galaxy halo becomes massive enough that accretion shocks develop, interrupting the cold streams of gas needed to replenish the disk (Dekel \& Birnboim 2006). Increasing AGN feedback from a growing supermassive black hole may shock or eject gas from the galaxy disk, reducing its capacity to form stars (Hopkins et al. 2006; Ogle et al. 2014). Ram-pressure stripping of the interstellar medium by the intercluster medium of a galaxy cluster can also remove cold gas (Sivanandam et al. 2014).

Studying the most massive spiral galaxies can give us clues as to which of the above evolutionary processes are primarily responsible for converting star-forming disk galaxies into redand-dead lenticulars or ellipticals. The existence of rapidly starforming, massive spirals with $M_{\text {stars }}>10^{11} M_{\odot}$ indicates that disk galaxies can postpone this fate under special circumstances. We present here the most optically luminous and biggest spiral galaxies at redshift $z<0.3$, found by mining the NASA/IPAC Extragalactic Database (NED). We assume a cosmology with $H_{0}=70, \Omega_{m}=0.3$, and $\Omega_{\Lambda}=0.7$ for computing all linear sizes and luminosities.

\section{SAMPLE}

This project is an offshoot of our work to determine the completeness of NED and explore its potential for systematic studies of galaxy populations (P. Ogle et al. 2016, in 
preparation). NED provides a unique fusion of multiwavelength photometry from Galaxy Evolution Explorer $(G A L E X)$, Sloan Digital Sky Survey (SDSS), and the 2-Micron All-Sky Survey (2MASS), among others, which we augment by Wide-field Infrared Survey Explorer (WISE) photometry, that allows us to estimate stellar masses and SFRs. We compared the redshift distribution of galaxies in NED at $z<0.3$ to a model redshift distribution for the universe derived using a redshift-independent luminosity function, in order to estimate the spectroscopic completeness of NED. We used the Schechter (1976) luminosity function fits of Blanton et al. (2003), which are based on $\sim 150,000$ SDSS galaxies as our benchmark. We adopt their characteristic absolute magnitude value of $M^{*}-5 \log _{10} h=$ $-20.44 \pm 0.01\left(L^{*}=5.41 \times 10^{43} \mathrm{erg} \mathrm{s}^{-1}\right.$ at $\left.6200 \AA\right)$ for the SDSS $r$-band luminosity function. The redshift limit was made large enough to capture the rarest, most luminous galaxies, but not so large as to require consideration of redshift evolution in the luminosity function.

\subsection{SDSS r-band Selection of the Most Optically Luminous Galaxies}

SDSS is the largest source of spectroscopic redshifts, with a spectroscopic selection limit of $r=17.77$ (Strauss et al. 2002). We find that NED is complete over the SDSS footprint out to $z=0.3$ for galaxies with $L_{r}>8 L^{*}$, the most optically luminous and massive galaxies in the low-redshift universe. Our sample is chosen from the 797,729 galaxies (type $=\mathrm{G}$ ) in NED with spectroscopic redshifts $z<0.3$, in the SDSS footprint, and detected in SDSS $r$ band. We apply Galactic extinction corrections (tabulated by NED) and K-corrections to the $r$ band magnitudes prior to making our sample selection. We find 1616 galaxies with redshift $z<0.3$ and luminosity $L_{r}>8 L^{*}$, which constitute our Ogle et al. Galaxy Catalog (OGC). The most luminous galaxy in the OGC is a $20 L^{*}$ elliptical brightest cluster galaxy (BCG).

\subsection{UV Selection Method for Super Spiral Galaxies}

We make a further selection for UV emission because we are interested in finding the most massive, actively star-forming disk galaxies. We recently matched and integrated the GALEX All-Sky Survey Catalog (GASC) and GALEX Medium Sky Survey Catalog (GMSC) with NED, using an automated, statistical algorithm (Ogle et al. 2015). We inspected the SDSS images of all 196 galaxies from the OGC that are detected in the GALEX NUV band (the OGC-UV subsample). Of these, we find 46 NUV-detected, $L_{r}>8 L^{*}$ galaxies with spiral morphology (Table 1). The remaining NUV sources include 118 ellipticals, 11 galaxies with $\mathrm{E}+\mathrm{A}$ spectra, 2 quasi-stellar objects (QSOs) with extended emission, and 19 galaxies with erroneous redshifts or magnitudes. The most luminous elliptical galaxy in OGC-UV is a $16 L^{*} \mathrm{BCG}$, while the most luminous spiral galaxy has $L_{r}=14 L^{*}$.

\subsection{Morphological Selection Method for Super Spiral Galaxies}

We inspected the 310 brightest galaxies of the full OGC sample, those with $L_{r}>10.5 L^{*}$, to see if we are missing any spirals with UV selection. Of these, we classified 11 super spirals, 253 ellipticals, 38 galaxies with erroneous redshifts or magnitudes, 6 lenticulars, 1 irregular, and $1 \mathrm{E}+\mathrm{A}$ galaxy. This inventory includes 7 additional super spirals (Table 1) that are not in the OGC-UV sample. Of these, 4 have no GALEX sources nearby, and 3 others have nearby GALEX sources that should be matched in NED, but are not, possibly because of confusion. This shows that our NUV selection, while relatively efficient (47/196) compared to morphological selection (11/ 310 ), leads to an incomplete sample, with only 4/11 super spirals recovered in this luminosity range. Part of the incompleteness $(3 / 11)$ is owing to incomplete matching of GALEX with NED, while the rest (4/11) may be attributed to the GALEX detection limit or coverage.

\section{PHOTOMETRY}

We conduct our investigation of super spirals primarily with photometry compiled by NED. We use SDSS DR6 $u, g, r, i, z$ photometry measured with the CModel method, which combines exponential plus deVaucouleurs model fitting. GALEX FUV and NUV photometry is taken from the GASC and GMSC, measured within a Kron elliptical aperture. We use 2MASS $J, H, K$ s total magnitudes from the 2MASS Extended Source Catalog (2MASX). NED objects are matched to All WISE using the Gator tool in the Infrared Science Archive (IRSA). We use AllWISE 4.6 and $12 \mu \mathrm{m}$ photometry within the largest available fixed-radius aperture of 24 !. 75 , which is wellmatched to the largest galaxy in our sample, with semimajor axis $a=24$ ". 5 .

\section{BASIC PROPERTIES OF SUPER SPIRALS}

\subsection{Optical and Mid-IR Colors}

The SDSS and WISE colors of super spirals lie along the blue sequence, similarly to less luminous star-forming disk galaxies (Figure 1(a)). The SDSS comparison sample is adopted from Alatalo et al. (2014), who show that LTGs and ETGs classified by Galaxy Zoo (GZ; Lintott et al. 2008) are well-separated in WISE [4.6]-[12] versus SDSS $u-r$ color space. The WISE [4.6]-[12] color ranges from 2.0 to 4.2 , typical of polycyclic aromatic hydrocarbon (PAH) and warm dust emission from gas-rich, actively star-forming galaxies. The $u-r$ color ranges from 1.4 to 4.4 , indicating star-forming disks with a range of SSFR or dust extinction. We estimate differential K-corrections of $\Delta(u-r)<0.2 \mathrm{mag}$ in the redshift range $z=0.1-0.3$, by convolving several spectral energy distribution (SED) models (e.g., those in the Appendix) with the SDSS filter curves. These corrections are not large enough to explain the additional scatter in the observed $u-r$ colors of super spirals.

There is a shift in the locus of super spiral colors compared to less-massive blue sequence galaxies. Super spirals tend to have redder $u-r$ and bluer [4.6]-[12] colors compared to the SDSS distribution. This could in principle indicate either lower SSFR or increased extinction. However, the high SSFR of our sample (Figure 1(b)) runs contrary to the first explanation. Six super spirals have $u-r>3.0$, a value not attained by less luminous SDSS LTGs. The two reddest galaxies (SS 53 and SS 09) may be misclassified peculiar elliptical galaxies with prominent shells. CGCG 122-067 (SS 50) may be redder because of its double bulge. The other 4 are clearly spirals, and require further investigation and custom photometry to determine the cause of their unusually red $u-r$ colors. 
Table 1

OGC Super Spiral Sample

\begin{tabular}{|c|c|c|c|c|c|c|c|c|c|c|c|}
\hline SS & OGC & NED Name & $L_{r}\left(L^{*}\right)$ & $D^{\mathrm{a}}$ & $M_{\text {stars }}^{\mathrm{b}}$ & $\mathrm{SFR}^{\mathrm{c}}$ & Redshift $^{\mathrm{d}}$ & NUV & $r$ & $u-r$ & Notes \\
\hline 01 & 0065 & 2MASX J10301576-0106068 & 13.9 & 81.3 & 11.25 & 1.54 & 0.28228 & $21.02^{\mathrm{e}}$ & 16.92 & 2.54 & bar \\
\hline 02 & 0073 & 2MASX J10405643-0103584 & 13.4 & 82.2 & 11.39 & 0.97 & 0.25024 & 21.65 & 16.64 & 2.16 & BCG \\
\hline 03 & 0139 & 2MASX J16394598+4609058 & 12.0 & 134. & 11.05 & 1.48 & 0.24713 & 19.85 & 16.63 & 2.37 & edge-on \\
\hline 04 & 0170 & 2MASX J10100707+3253295 & 11.6 & 87.1 & 11.27 & 1.40 & 0.28990 & 20.14 & 17.10 & 2.68 & BCG, bar \\
\hline 05 & 0217 & 2MASX J13275756+3345291 & 11.2 & 68.8 & 11.05 & 1.81 & 0.24892 & 19.44 & 16.72 & 2.02 & starburst, bar \\
\hline 06 & 0256 & 2MASX J11593546+1257080 & 10.9 & 87.2 & 10.89 & 1.26 & 0.26353 & 20.04 & 16.95 & 1.79 & $\cdots$ \\
\hline 07 & 0265 & SDSS J115052.98+460448.1 & 10.8 & 88.1 & 10.94 & $<0.74$ & 0.28946 & $>21.51^{\mathrm{e}}$ & 17.19 & 3.25 & faint spiral \\
\hline 08 & 0290 & 2MASX J12343099+5156295 & 10.6 & 62.4 & 11.13 & 1.71 & 0.29592 & 19.57 & 17.25 & 1.67 & Sy1, asymm. \\
\hline 09 & 0299 & 2MASX J09094480+2226078 & 10.5 & 83.1 & 11.26 & $<1.15$ & 0.28539 & 21.40 & 17.25 & 3.73 & BCG, shells? \\
\hline 10 & 0302 & 2MASX J15430777+1937522 & 10.5 & 65.5 & 11.37 & $2.45^{\mathrm{f}}$ & 0.22941 & $\ldots$ & 17.07 & 0.40 & QSO, tidal arm \\
\hline 11 & 0306 & SDSS J122100.48+482729.1 & 10.5 & 75.0 & 10.82 & 1.02 & 0.29966 & 20.15 & 17.29 & 1.69 & $\cdots$ \\
\hline 12 & 0345 & 2MASX J09260805+2405242 & 10.3 & 81.2 & 11.27 & 1.38 & 0.22239 & 19.61 & 16.57 & 3.38 & BCG, face-on \\
\hline 13 & 0388 & 2MASX J17340613+6029190 & 10.1 & 63.5 & 11.20 & 1.27 & 0.27596 & 20.51 & 17.19 & 2.71 & BGG \\
\hline 14 & 0441 & SDSS J095727.02+083501.7 & 9.9 & 87.6 & 11.53 & 1.13 & 0.25652 & 20.88 & 16.99 & 2.19 & $\cdots$ \\
\hline 15 & 0454 & 2MASXi J1003568+382901 & 9.9 & 56.4 & 10.82 & 1.65 & 0.25860 & 19.79 & 16.97 & 1.70 & starburst \\
\hline 16 & 0543 & 2MASX J09470010+2540462 & 9.6 & 99.3 & 11.07 & 1.13 & 0.10904 & 17.74 & 14.83 & 2.57 & bar, Sy1? \\
\hline 17 & 0586 & 2MASX J11535621+4923562 & 9.5 & 90.2 & 11.11 & 1.43 & 0.16673 & 19.92 & 15.90 & 2.64 & $\mathrm{BCG}, \mathrm{Sy} 2$ \\
\hline 18 & 0595 & 2MASX J07550424+1353261 & 9.5 & 76.6 & 11.12 & 1.30 & 0.22264 & 19.71 & 16.67 & 2.47 & bar \\
\hline 19 & 0696 & SDSS J102154.85+072415.5 & 9.2 & 69.7 & $<11.57$ & 1.35 & 0.29061 & 19.89 & 17.37 & 2.01 & $\cdots$ \\
\hline 20 & 0713 & 2MASX J08265512+1811476 & 9.2 & 81.9 & 11.27 & 1.32 & 0.26545 & 21.01 & 17.16 & 3.26 & bar \\
\hline 21 & 0755 & SDSS J113800.88+521303.9 & 9.1 & 63.9 & 10.76 & 1.14 & 0.29593 & 20.94 & 17.41 & 2.12 & ring \\
\hline 22 & 0789 & 2MASX J08542169+0449308 & 9.0 & 86.0 & 10.96 & 1.30 & 0.15679 & 18.68 & 15.83 & 2.49 & 2 spirals, bar \\
\hline 23 & 0799 & 2MASX J10472505+2309174 & 9.0 & 72.2 & 11.12 & 1.20 & 0.18256 & 20.61 & 16.19 & 2.68 & bar \\
\hline 24 & 0800 & 2MASX J11191739+1419465 & 9.0 & 70.8 & 10.93 & 1.15 & 0.14377 & 18.75 & 15.57 & 2.39 & $\cdots$ \\
\hline 25 & 0804 & SDSS J135546.07+025455.8 & 9.0 & 84.2 & $<11.35$ & 1.01 & 0.23884 & 19.77 & 16.87 & 1.74 & $\cdots$ \\
\hline 26 & 0830 & SDSS J141754.96+270434.4 & 9.0 & 68.6 & 10.70 & 1.11 & 0.15753 & 19.74 & 15.79 & 2.86 & $\cdots$ \\
\hline 27 & 0926 & 2MASX J10304263+0418219 & 8.8 & 72.7 & 11.19 & 1.60 & 0.16902 & 19.08 & 15.93 & 2.16 & $\cdots$ \\
\hline 28 & 0928 & 2MASX J12374668+4812273 & 8.8 & 66.0 & 11.01 & 1.57 & 0.27245 & 19.79 & 17.24 & 2.10 & $\cdots$ \\
\hline 29 & 0975 & 2MASX J11410001+3848078 & 8.7 & 72.1 & 11.08 & 1.38 & 0.26770 & 20.79 & 17.21 & 2.15 & $\cdots$ \\
\hline 30 & 0983 & SDSS J153618.97+452246.8 & 8.7 & 80.2 & 10.48 & 1.07 & 0.23618 & 20.15 & 16.85 & 2.13 & $\cdots$ \\
\hline 31 & 1046 & 2MASX J09362208+3906291 & 8.6 & 69.6 & 10.99 & 1.37 & 0.28293 & 20.09 & 17.36 & 1.78 & $\cdots$ \\
\hline 32 & 1088 & SDSS J140138.37+263527.6 & 8.5 & 78.2 & $<11.50$ & 1.24 & 0.28396 & 19.99 & 17.38 & 2.08 & $\cdots$ \\
\hline 33 & 1196 & SDSS J154950.91+234444.1 & 8.4 & 69.3 & $<11.35$ & 1.30 & 0.26208 & 20.48 & 17.27 & 2.02 & $\cdots$ \\
\hline 34 & 1250 & 2MASX J12321515+1021195 & 8.3 & 71.4 & 10.95 & 1.06 & 0.16588 & 19.69 & 16.04 & 2.76 & 2 bulges? \\
\hline 35 & 1268 & 2MASX J12005393+4800076 & 8.3 & 62.7 & 11.10 & 1.45 & 0.27841 & 20.04 & 17.37 & 2.05 & $\mathrm{BCG}$ \\
\hline 36 & 1273 & 2MASX J07380615+2823592 & 8.3 & 76.6 & 11.01 & 1.28 & 0.23091 & 20.13 & 16.92 & 2.34 & $\cdots$ \\
\hline 37 & 1304 & 2MASX J16014061+2718161 & 8.3 & 82.3 & 11.03 & 1.17 & 0.16440 & 17.60 & 16.06 & 1.60 & BCG, 2 spirals \\
\hline 38 & 1312 & SDSS J143447.86+020228.6 & 8.2 & 75.4 & 10.67 & 1.48 & 0.27991 & 20.43 & 17.42 & 2.24 & $\cdots$ \\
\hline 39 & 1323 & SDSS J112928.74+025549.9 & 8.2 & 69.7 & 10.63 & 1.23 & 0.23960 & 19.56 & 17.01 & 2.00 & $\cdots$ \\
\hline 40 & 1352 & SDSS J101603.97+303747.9 & 8.2 & 68.8 & 10.73 & $\ldots g$ & 0.25191 & 21.16 & 17.13 & 2.94 & $\cdots$ \\
\hline 41 & 1375 & 2MASX J00155012-1002427 & 8.2 & 68.4 & 10.94 & 0.91 & 0.17601 & $\cdots$ & 16.23 & 2.09 & $\cdots$ \\
\hline 42 & 1395 & 2MASX J13103930+2235023 & 8.1 & 65.6 & 11.08 & 1.15 & 0.23123 & 19.91 & 16.87 & 2.59 & $\cdots$ \\
\hline 43 & 1420 & 2MASX J13475962+3227100 & 8.1 & 87.5 & 10.94 & 1.23 & 0.22306 & 20.25 & 16.79 & 2.37 & BGG \\
\hline 44 & 1464 & 2MASX J10041606+2958441 & 8.1 & 57.4 & 11.04 & 1.81 & 0.29844 & 20.64 & 17.59 & 2.06 & starburst \\
\hline 45 & 1500 & 2MASX J10095635+2611324 & 8.1 & 63.7 & 10.98 & 1.33 & 0.24089 & 19.99 & 17.03 & 2.19 & $\cdots$ \\
\hline 46 & 1501 & 2MASX J09334777+2114362 & 8.1 & 63.6 & 11.00 & 1.69 & 0.17219 & 17.84 & 16.17 & 1.60 & QSO, 2 nuclei \\
\hline 47 & 1544 & 2MASX J14472834+5908314 & 8.0 & 68.4 & 11.13 & 1.13 & 0.24551 & 20.22 & 17.04 & 2.15 & $\cdots$ \\
\hline 48 & 1546 & 2MASX J13435549+2440484 & 8.0 & 60.7 & 11.05 & 0.89 & 0.13725 & 19.54 & 15.56 & 2.50 & $\cdots$ \\
\hline 49 & 1554 & 2MASX J13422833+1157345 & 8.0 & 57.1 & 11.08 & 1.43 & 0.27873 & 21.66 & 17.43 & 2.20 & $\cdots$ \\
\hline 50 & 1559 & CGCG 122-067 & 8.0 & 81.4 & 11.13 & 1.00 & 0.08902 & 18.27 & 14.56 & 3.13 & BCG, 2 bulges \\
\hline 51 & 1606 & SDSS J121644.34+122450.5 & 8.0 & 77.9 & $<11.31$ & 1.13 & 0.25694 & 20.12 & 17.22 & 1.76 & bar, Sy1 \\
\hline 52 & 1608 & SDSS J040422.91-054134.9 & 8.0 & 79.5 & 10.56 & 1.07 & 0.25055 & 20.38 & 17.27 & 2.37 & flocculent \\
\hline 53 & 1611 & 2MASX J00380781-0109365 & 8.0 & 83.9 & 11.31 & 0.91 & 0.20828 & 21.33 & 16.65 & 4.38 & E with shells? \\
\hline
\end{tabular}

Notes.

a Isophotal diameter (kpc) at $r=25.0 \mathrm{mag} \operatorname{arcsec}^{-2}$.

${ }^{\mathrm{b}} \log _{10} M_{\text {stars }}\left(M_{\odot}\right)$ or $3 \sigma$ upper limit, based on 2MASS $K$ s luminosity and $u-r$ color.

${ }^{\mathrm{c}} \log _{10}$ SFR $\left(M_{\odot} \mathrm{yr}^{-1}\right)$ or $95 \%$ confidence upper limit, based on WISE $12 \mu \mathrm{m}$ luminosity.

${ }^{\mathrm{d}}$ SDSS DR9 redshift.

e GALEX NUV-band photometry measured in 14" aperture. Source not in GASC or GMSC.

${ }^{f}$ This SFR may be overestimated by a large factor because of the QSO nucleus.

${ }^{\mathrm{g}}$ WISE data compromised by nearby IR-bright star. 

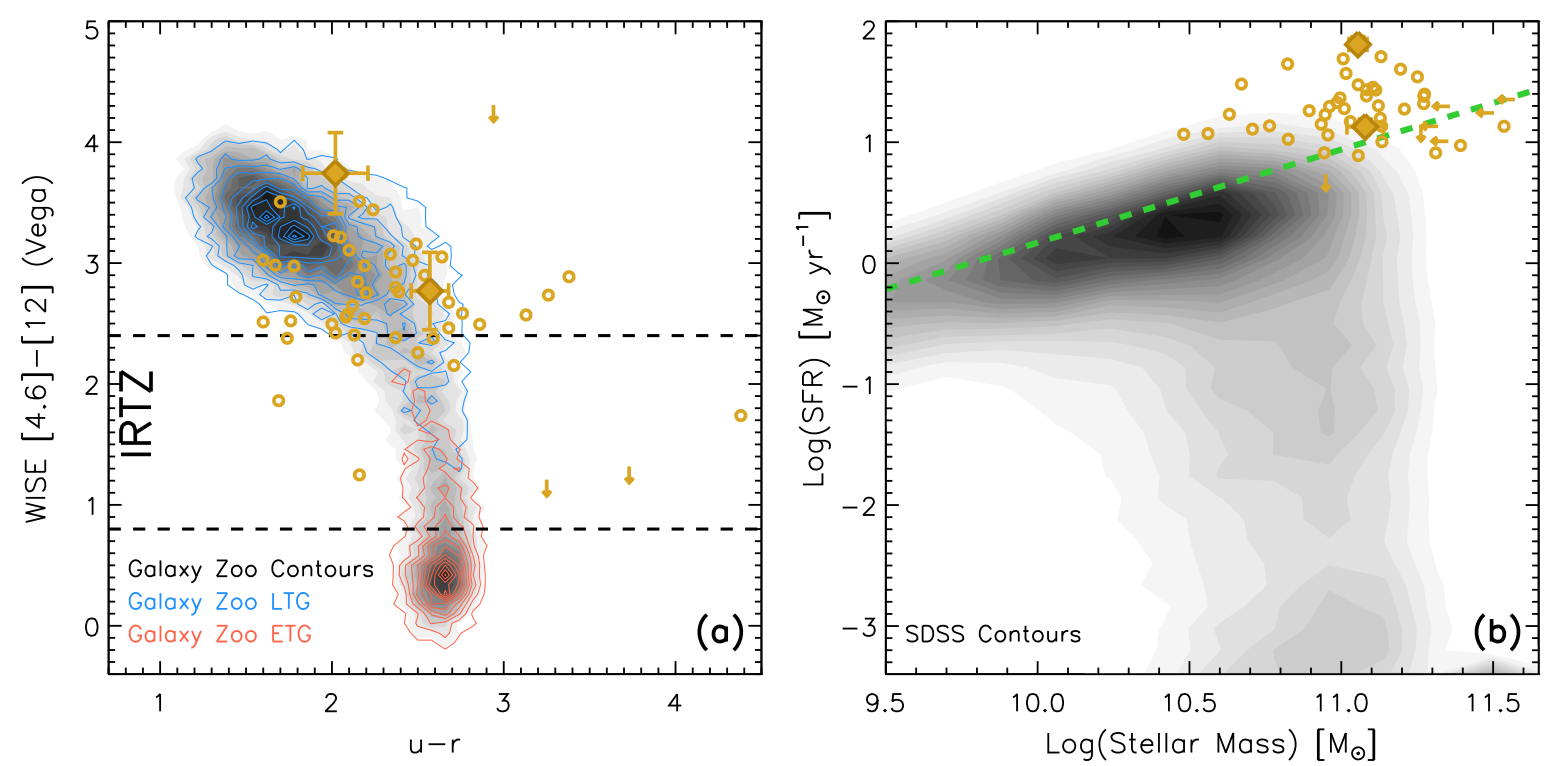

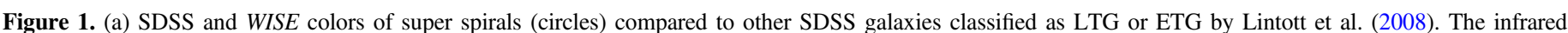

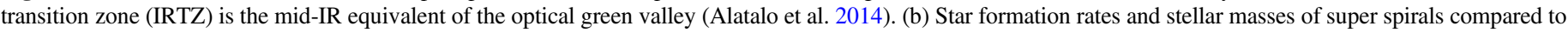

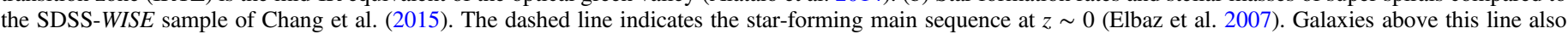

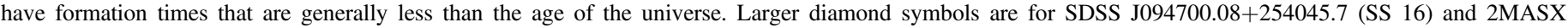
J13275756+334529 (SS 05), with detailed SED analysis presented in the Appendix.

\subsection{Stellar Mass and SFR}

We estimate stellar mass from 2MASS Ks luminosity together with an SDSS $u-r$ color-dependent mass-to-light ratio estimated using the prescription of Bell et al. (2003), giving $M_{\text {stars }} / L=0.75-1.34 \quad M_{\odot} / L_{\odot}$. We apply a small correction to the stellar masses to convert to a Chabrier initial mass function. This yields stellar masses that are consistent with more sophisticated SED template fitting (Appendix). We find stellar masses in the range $M=0.3-3.4 \times 10^{11} M_{\odot}$.

We estimate the SFR from the WISE $12 \mu \mathrm{m}$ luminosity using the prescription of Chang et al. (2015), which was established by SED-fitting more than 630,000 SDSS galaxies with MAGPHYs (da Cunha et al. 2008). While accurate for starforming galaxies, this method may overestimate the starformation rate for early type galaxies where dust may be heated by other sources not directly related to star formation, or in the presence of a luminous AGN. We further validate our WISE single-band SFRs against MAGPHYS SED-fitting for two representative super spirals (Appendix). The WISE $12 \mu \mathrm{m}$ monochromatic luminosities of super spirals range from $0.3-3.5 \times 10^{44} \mathrm{erg} \mathrm{s}^{-1}\left(0.8-9.8 \times 10^{10} L_{\odot}\right)$, corresponding to SFRs of 5-65 $M_{\odot} \mathrm{yr}^{-1}$.

We compare our sample to the SDSS-WISE sample of Chang et al. (2015), who estimated SFR and $M_{\text {stars }}$ with MAGPHYs. We find that most super spirals lie well above an extrapolation of the SFMS to higher mass (Figure 1(b)). This is a region of the SFR versus mass diagram that is very sparsely populated. The vast majority of SDSS disk galaxies in this mass range have significantly lower SFR and SSFR.

Our $r$-band luminosity plus NUV detection criteria tend to select galaxies with high global SFRs. However, the SDSS spectra reveal a relatively old bulge stellar population for most super spirals. We do find an indication of starburst activity in the SDSS bulge spectra of 3 super spirals (SS 05-see the Appendix, SS 15, and SS 44) with strong young stellar population contributions and high-equivalent width $\mathrm{H} \alpha$ emission. These three galaxies also have relatively blue SDSS $u-r$ colors and red WISE [4.6]-[12] colors, both indicative of a high global SSFR.

\subsection{Active Galactic Nuclei}

The super spiral galaxies in our sample contains 3 Seyfert 1 nuclei and 2 QSOs with broad Balmer lines and strong [O $\mathrm{III}]$ in their SDSS spectra (Table 1). There is also 1 Seyfert 2 nucleus with strong [O III] but narrow Balmer lines. There is likely a dominant contribution from the QSO to the IR luminosity of 2MASX J15430777+1937522 (SS 10), which has the greatest WISE $12 \mu \mathrm{m}$ luminosity of our sample $\left(1.7 \times 10^{45} \mathrm{erg} \mathrm{s}^{-1}\right.$ or $\left.4.3 \times 10^{11} L_{\odot}\right)$. The two QSOs are also detected at X-ray wavelengths by ROSAT. One additional galaxy (2MASX $\mathrm{J} 10095635+2611324=\mathrm{SS} 45$ ) is detected in X-rays, but has no obvious signature of an AGN in its SDSS nuclear spectrum. There is so far no indication of any extended X-ray emission associated with super spirals, though none have been specifically targeted for this. It will be important to make deep $\mathrm{X}$-ray observations of super spirals to quantify any X-ray halo emission in comparison to giant elliptical galaxies. Only two super spirals are detected by the NVSS radio survey (2MASX $\mathrm{J} 14472834+5908314=\mathrm{SS} 47$ and CGCG 122-067 = SS 50), but the resolution is insufficient to distinguish between radio emission from star formation activity or from a radio jet. The presence of luminous AGNs in $11 \%$ of super spirals indicates that they are continuing to grow their supermassive black holes. It is imperative to measure the distribution of bulge and supermassive black hole masses in our super spiral sample to see if they follow the same relation as lower-mass spiral bulges.

\subsection{Size, SFR Surface Density, and Morphology}

The sizes of super spirals range from 57 to $134 \mathrm{kpc}$, with a median size of $72 \mathrm{kpc}$, using the SDSS DR6 $r$-band isophotal diameter at $25.0 \mathrm{mag} \operatorname{arcsec}^{-2}$ (Table 1 and Figure 2). Their deprojected SFR surface densities range from $1.5 \times 10^{-3}$ to 


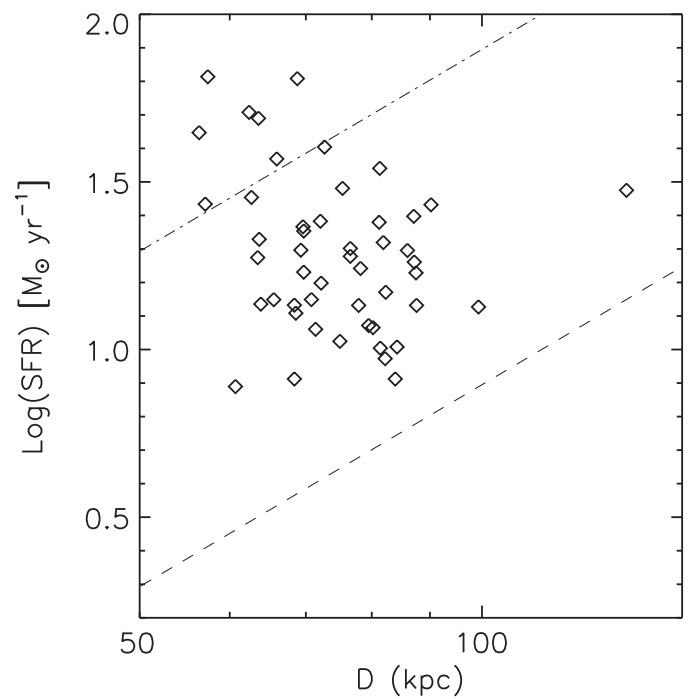

Figure 2. Super spirals range in diameter from 57 to $134 \mathrm{kpc}$. The dashed and dot-dashed lines indicate deprojected SFR surface densities of $1 \times 10^{-3}$ and $1 \times 10^{-2} M_{\odot} \mathrm{yr}^{-1} \mathrm{kpc}^{-2}$, respectively.

$2.0 \times 10^{-2} M_{\odot} \mathrm{yr}^{-1} \mathrm{kpc}^{-2}$. A plot of SFR versus diameter shows considerable scatter (Figure 2). However, the five most rapidly star-forming galaxies, with $\log (\mathrm{SFR})>1.6$, all have diameters $D<70 \mathrm{kpc}$. The most MIR-luminous super spiral (SDSS J094700.08+254045.7, see the Appendix), also has the largest deprojected SFR surface density. The largest super spiral, 2MASX J16394598+4609058 (SS 03, Figure 3), has a diameter of $134 \mathrm{kpc}$ and a relatively low SFR surface density of $2.0 \times 10^{-3} M_{\odot} \mathrm{yr}^{-1} \mathrm{kpc}^{-2}$.

Super spirals display a range of morphologies, from flocculent to grand-design spiral patterns (Figure 4). At least 9 super spirals have prominent stellar bars visible in the SDSS images (Table 1: Notes). There are morphological peculiarities in several cases, including one-arm spirals, multi-arm spirals, rings, and asymmetric spiral structure (Figure 4 and Table 1). These types of features may indicate past or ongoing galaxy mergers or collisions.

\subsection{Bulge-disk Decomposition}

We make use of the bulge-disk decompositions of Simard et al. (2011) to quantify the relative contributions of the bulge and disk to the luminosity of super spirals (Table 2). The galaxy $g$ and $r$ band SDSS images are jointly fit by a de Vaucouleurs profile for the bulge (Sérsic index $n_{b}=4$ ), plus an exponential disk. We compare super spirals to a representative subsample of 4686 spiral galaxies with $z>0.09$ classified by Galaxy Zoo (GZ, Figure 5), with bulge-disk decompositions also by Simard et al. (2011). We find a much narrower distribution of $r$-band bulge to total luminosity $(B / T)$ for super spirals, with a median value of $B / T=0.17$, and a deficit of $B / T$ values $<0.1$. A Kolmogorov-Smirnoff $(\mathrm{K}-\mathrm{S})$ test shows that the distributions differ significantly, with a probability of only 0.0027 that super spirals are drawn from the same population as GZ spirals. The lack of super spirals with $B / T<0.1$ may be consistent with a past history of significant merger activity. The bulge ellipticity distribution of super spirals is not significantly different from that of GZ spirals (Figure 5(c)). We note that since the profile fits do not include a bar component, the

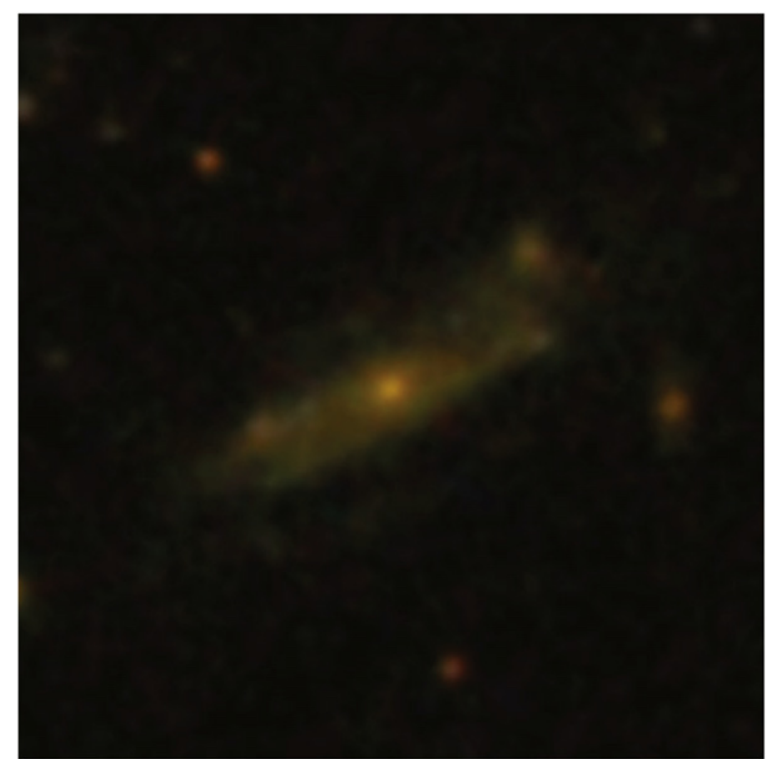

Figure 3. Largest super spiral galaxy, with $L_{r}=12 L^{*}$ and an isophotal diameter of $134 \mathrm{kpc}, 2 \mathrm{MASX}$ J16394598+4609058 (SS 03, $z=0.24713$ ). The SDSS image is $50.77(197 \mathrm{kpc})$ on each side.

ellipticities may be augmented by the presence of a bar or double bulge.

The disk inclination distribution of super spirals also differs significantly from that of GZ spirals, and from the expected $\sin (i)$ dependence (Figure 5(b)). A K-S test gives a probability of $\ll 0.001$ that super spirals and GZ spirals are drawn from the same inclination distribution. Only $5(9 \%)$ of super spirals have inclinations of $i>60^{\circ}$, compared to the expectation of $50 \%$ for randomly oriented disks. This indicates that we are missing roughly $45 \%$ of the super spirals in our luminosity range, possibly because of internal extinction at the NUV selection wavelength. The GZ spiral inclination distribution also differs from the expectation for randomly oriented disks, with an excess at inclinations $>60^{\circ}$ that may reveal a bias for GZ to classify edge on disks as spirals or to misclassify edge-on lenticulars as spirals.

The median disk exponential scale length of super spirals is $12.2 \mathrm{kpc}, 2.3$ times as large as the $5.3 \mathrm{kpc}$ median for GZ spirals, confirming the giant disk sizes of super spirals (Figure $5(\mathrm{~d})$ ). A K-S test gives a probability of $\ll 0.001$ that super spirals and GZ spirals are drawn from the same size distribution. The galaxy smoothness parameter (Simard et al. 2009), which quantifies the fractional residuals to the model fit inside two half-light radii, is $S 2=0.02-0.24$ in $r$ band. The $B / T$ and $S 2$ parameters of bulge-disk decompositions have been used by others to quantitatively select early-type galaxies, with $B / T>0.35$ and $S 2<0.075$ as criteria (Simard et al. 2009). Several super spirals in our sample meet these criteria, but we are nevertheless confident of the detection of a significant spiral disk in most of these cases.

\section{GALAXY MERGER CANDIDATES}

We find four super spiral merger candidates with apparent double stellar bulges or double nuclei (Figure 6). The SDSS spectra only cover the dominant or central bulge or nucleus of each system. Spectroscopy of the secondary bulges or nuclei will be necessary to confirm or rule out these merger candidates as true physical pairs or multiples. 


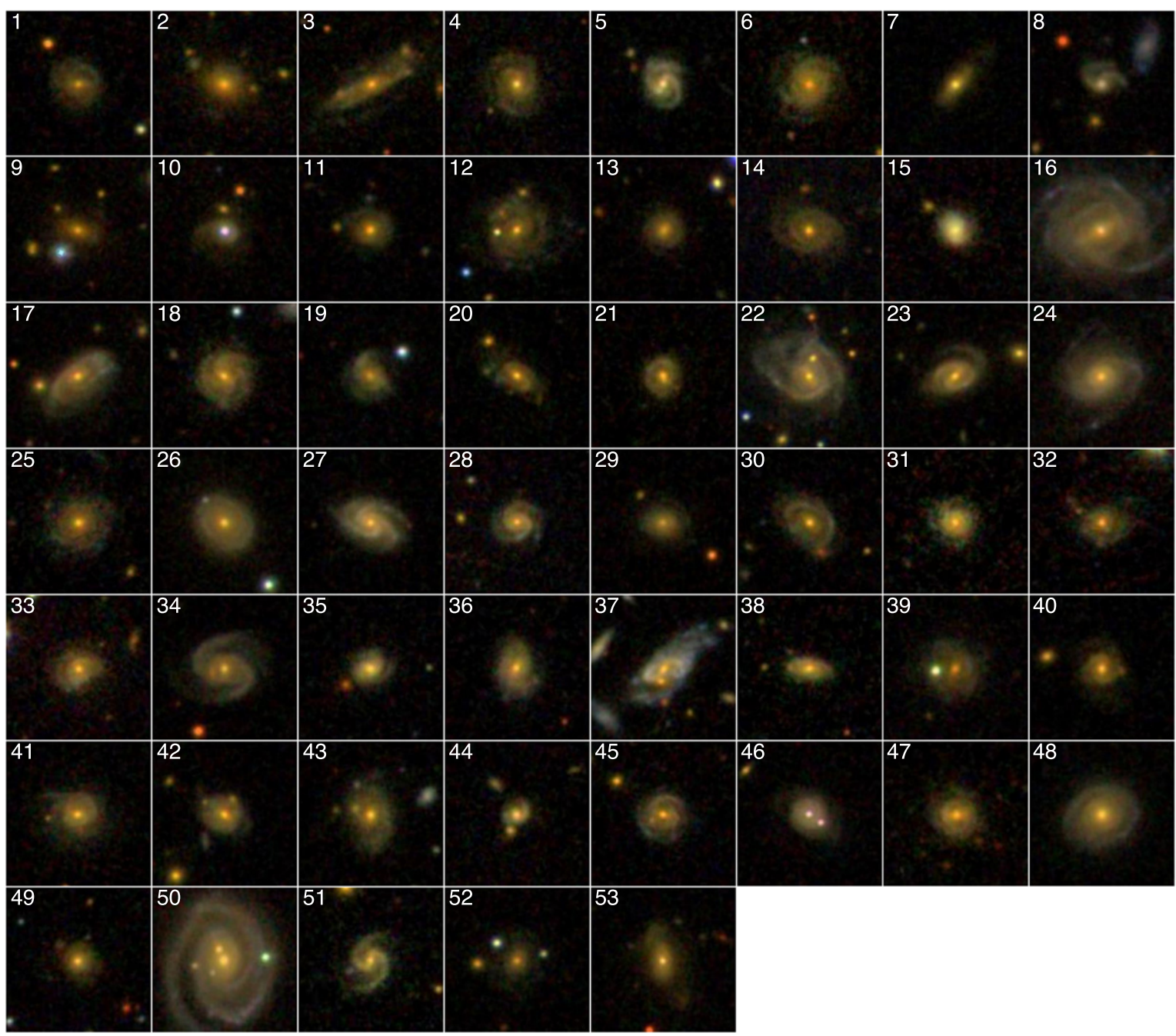

Figure 4. SDSS images of super spirals, 40" on a side. Examples with peculiar morphology: (1) multi-arm spiral, (8) asymmetric 2-arm spiral, (10) QSO host with tidal arm, (21) ring galaxy, (23) possible tidal arm, (33) asymmetric disk, (34) possible secondary bulge, (53) partial arms or shells.

The merger candidate 2MASX J08542169+0449308 (SS 22) appears to be a nearly equal mass major spiral pre-merger. The arms of both spirals are wound in the same direction, and the disks appear to be overlapping in the plane of the sky. The stretched out spiral arms of both spiral galaxy components, together with an apparent tidal arm at PA $=0^{\circ}$ (measured counterclockwise from North) suggest an ongoing tidal interaction.

The merger candidate 2MASX J16014061+2718161 (SS 37) is a BCG, surrounded by several other disk galaxy companions (Figures 6 and 7). The host cluster is identified as GMBCG J240.41924+27.30444, with a photometric redshift of 0.193 (Table 3). There are clear distortions to the spiral structures of both spiral galaxy components that appear to be involved in this merger.

The merger candidate 2MASX J09334777+2114362 (SS 46) appears to be a double AGN system. The primary, central nucleus is identified as an SDSS QSO. The secondary nucleus has similar flux and color to the primary nucleus, but it does not have an SDSS spectrum to confirm that it is a true physical double AGN. The galaxy disk has high surface brightness, suggestive of starburst activity. Faint outer spiral arms are also suggestive of a recent galaxy interaction.
The merger candidate and BCG CGCG 122-067 (SS 50) appears to be a late stage $\sim 2: 1$ major merger. The double bulge is surrounded by a common inner disk. Two giant spiral arms emerge from this central disk, one from each bulge, making a complete circuit around the disk. A large gap is seen between the arms at $\mathrm{PA}=0^{\circ}-90^{\circ}$. There are three other possible merging nuclei, including a bright green point source at $\mathrm{PA}=270^{\circ}$, that raise the possibility that this is a fivecomponent multiple merger system. Such multiple mergers are reminiscent of the elliptical nest galaxies that are sometimes found at the centers of galaxy clusters.

\section{ENVIRONMENT}

We checked NED for known galaxy clusters and groups within $1^{\prime}$ of each super spiral (Table 3). Seven of the super spirals are candidate BCGs, within $0 . " 8$ of a galaxy cluster. Two are candidate brightest group galaxies (BGGs), within $1^{\prime}$ of a compact galaxy group. Most of the clusters only have photometric redshifts and have yet to be verified spectroscopically. However, the photometric redshifts are all within $\Delta z=0.04$ of the super spiral spectroscopic redshift, which suggests a true physical association. The two associations of 
Table 2

Bulge-disk Decomposition (Simard et al. 2011)

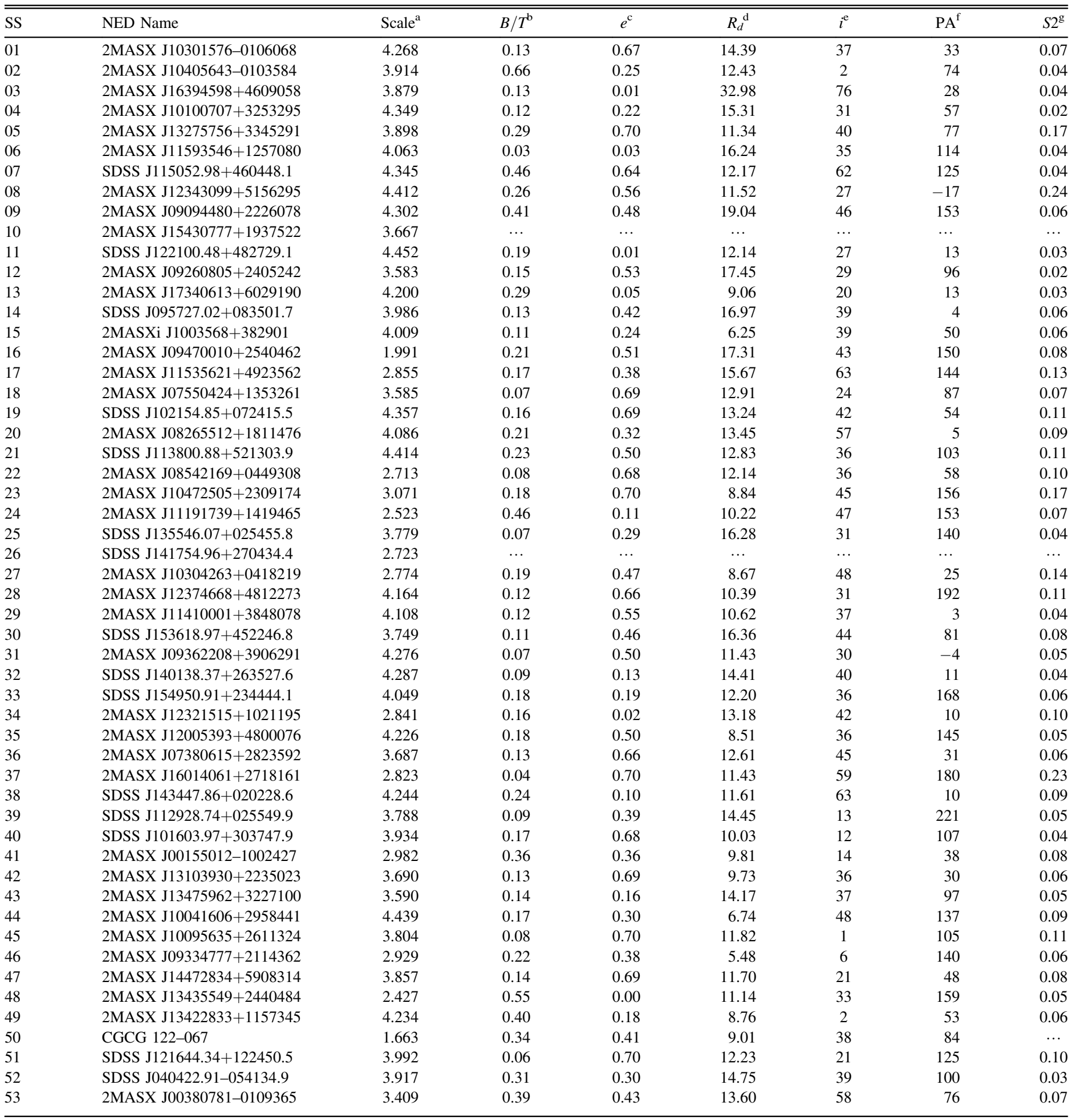

Notes.

a Scale [kpc/"].

${ }^{\mathrm{b}}$ Bulge fraction in SDSS DR7 $r$-band image.

${ }^{c}$ Bulge eccentricity.

${ }^{\mathrm{d}}$ Disk exponential scale length.

${ }^{\mathrm{e}}$ Disk inclination $\left({ }^{\circ}\right)$.

${ }^{\mathrm{f}}$ Disk PA $\left({ }^{\circ}\right)$

${ }^{\mathrm{g}}$ Smoothness in $r$ band. 

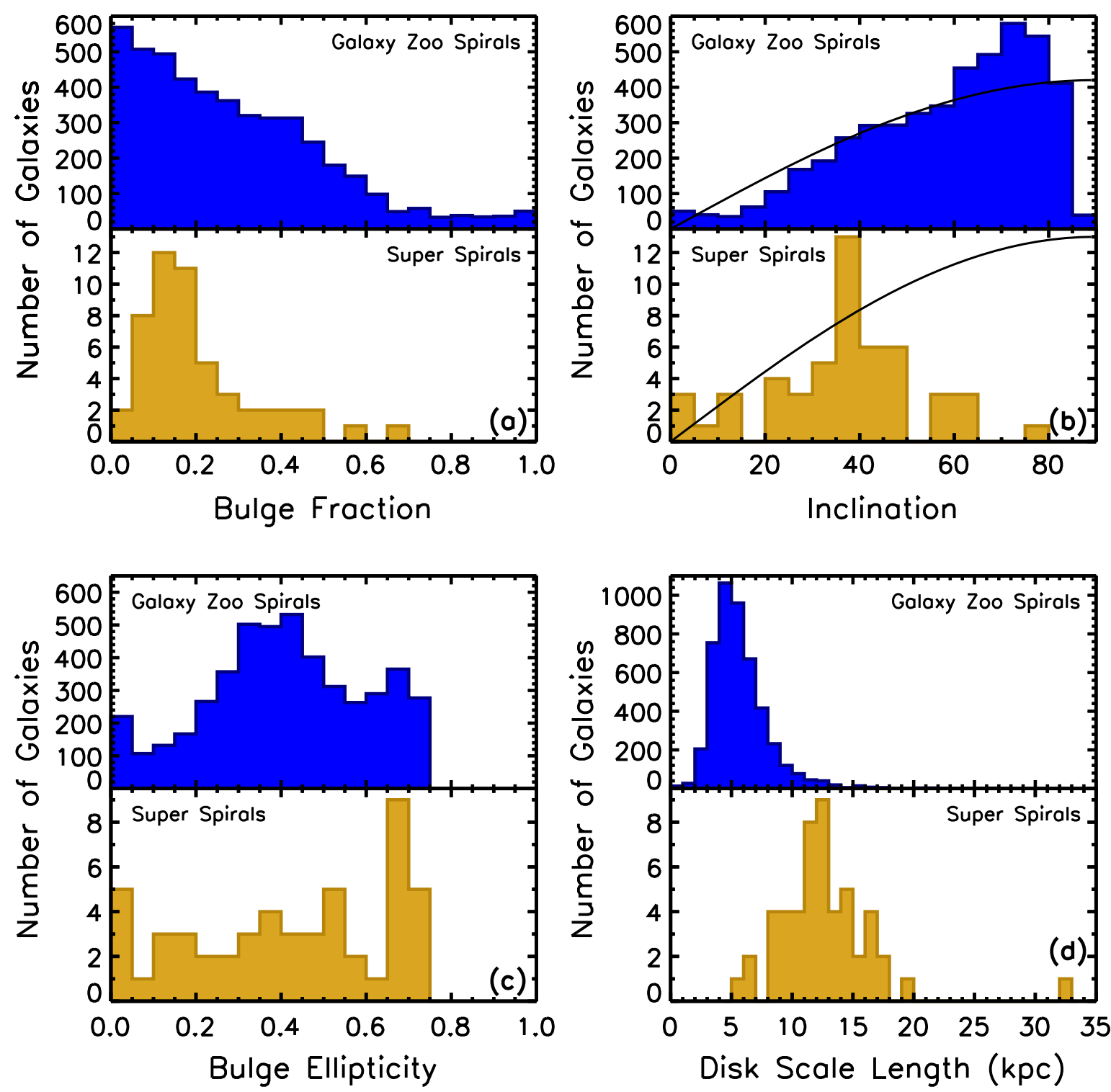

Figure 5. Distributions of super spiral and Galaxy Zoo spiral disk-bulge decomposition parameters, as measured by Simard et al. (2011): (a) Bulge to total (B/T) $r$-band luminosity fraction, (b) disk inclination distribution compared to the $\sin (i)$ expectation for randomly oriented disks (black curve), (c) bulge ellipticity, and (d) disk exponential scale length.
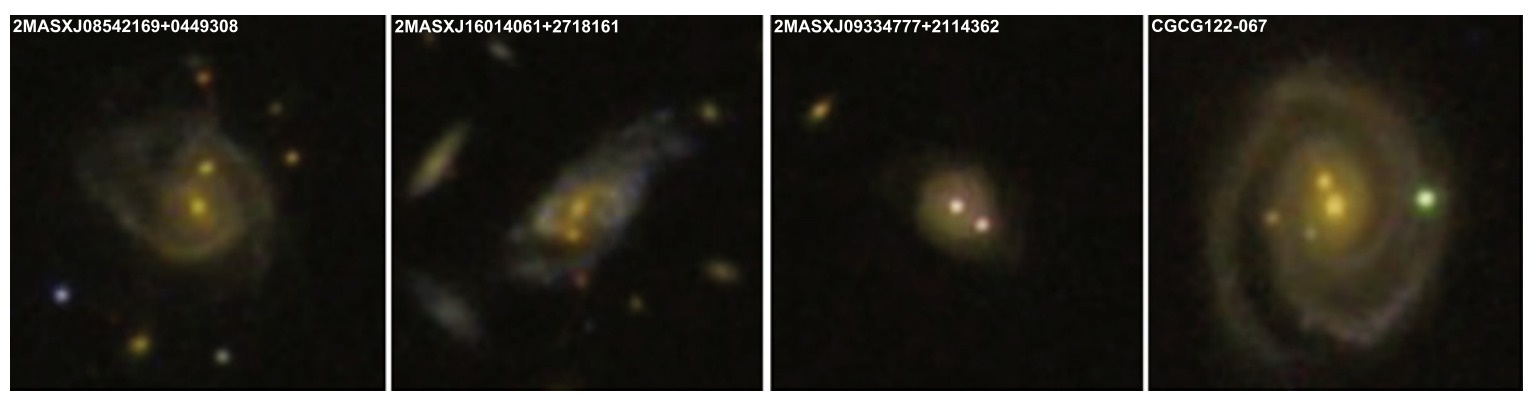

Figure 6. SDSS images of super spiral merger candidates. (a) Possible collision in progress of two spirals. (b) Possible collision or merger of two spirals, also a brightest cluster galaxy (see also Figure 7). (c) High-surface brightness disk with possible double AGN, with faint outer arms. The nucleus at the center is classified as an SDSS QSO. The second bright point source and possible AGN, near the edge of the disk, has a similar color to the primary AGN. (d) Possible late-stage major merger with two stellar bulges, with a striking grand spiral design surrounding both nuclei. Three other point sources may mark additional merging components or nuclei, reminiscent of nest galaxies commonly found at the centers of galaxy clusters. Each SDSS image is $48^{\prime \prime}$ on a side.

super spirals with compact groups are only based on their small angular separation, with no independent redshift available for the groups.
We used NED's Environment Tool to further explore the environments of the super spiral BCG and BGG candidates. This tool performs a redshift-constrained cone search for 


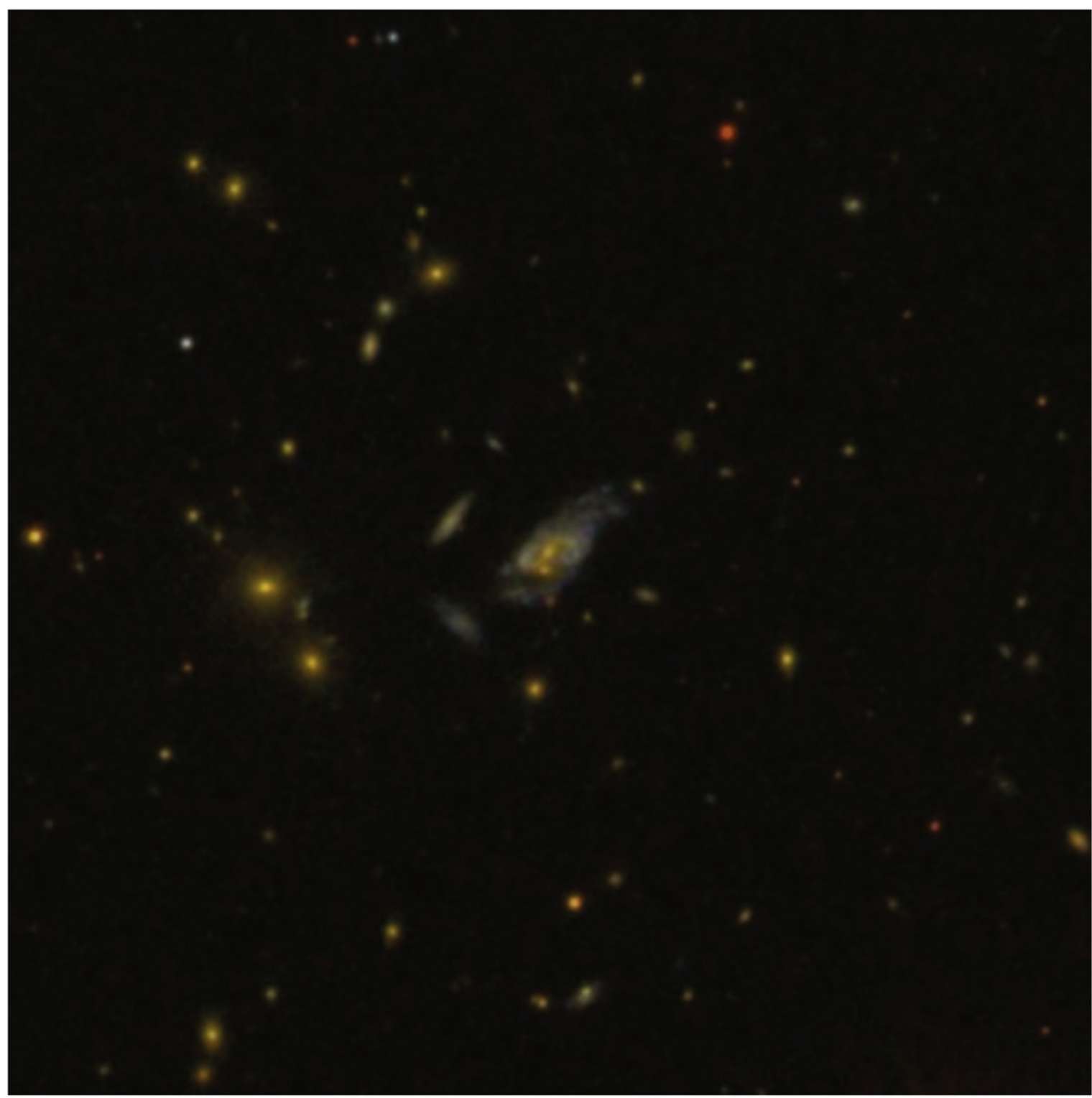

Figure 7. Super spiral merger candidate 2MASXJ16014061+2718161 (SS 37) is the brightest cluster galaxy of galaxy cluster GMBCG J240.41924+27.30444. The SDSS image is $203^{\prime \prime}(572 \mathrm{kpc})$ on each side.

Table 3

Candidate Cluster and Group Membership

\begin{tabular}{|c|c|c|c|c|c|c|c|c|c|}
\hline SS & NED Name & Redshift & $\mathrm{N} 1^{\mathrm{a}}$ & $\mathrm{N} 10^{\mathrm{b}}$ & Cluster Name & Type & Redshift & ztype $^{\mathrm{c}}$ & $\operatorname{Sep}\left({ }^{\prime}\right)$ \\
\hline 02 & 2MASX J10405643-0103584 & 0.250303 & 1 & 8 & SDSS CE J160.241898-01.069106 & GClstr & 0.254019 & EST & 0.013 \\
\hline 04 & 2MASX J10100707+3253295 & 0.289913 & 2 & 17 & GMBCG J152.52936+32.89139 & GClstr & 0.319000 & PHOT & 0.001 \\
\hline 09 & 2MASX J09094480+2226078 & 0.285386 & 1 & 9 & GMBCG J137.43670+22.43538 & GClstr & 0.303000 & PHOT & 0.000 \\
\hline 12 & 2MASX J09260805+2405242 & 0.222451 & 1 & 22 & WHL J092608.1+240524 & GClstr & 0.178000 & PHOT & 0.000 \\
\hline 13 & 2MASX J17340613+6029190 & 0.275807 & 1 & 2 & SDSSCGB 59704 & GGroup & $0.276000^{\mathrm{d}}$ & SPEC & 0.450 \\
\hline 17 & 2MASX J11535621+4923562 & 0.166892 & 3 & 69 & OGC 0586 CLUSTER & GClstr & 0.166187 & SPEC & 0.000 \\
\hline 35 & 2MASX J12005393+4800076 & 0.278617 & 1 & 13 & GMBCG J180.22479+48.00211 & GClstr & 0.252000 & PHOT & 0.001 \\
\hline 37 & 2MASX J16014061+2718161 & 0.164554 & 3 & 163 & GMBCG J240.41924+27.30444 & GClstr & 0.193000 & PHOT & 0.000 \\
\hline 43 & 2MASX J13475962+3227100 & 0.223113 & 1 & 13 & SDSSCGB 16827 & GGroup & $\ldots^{\mathrm{d}}$ & $\ldots$ & 0.748 \\
\hline 50 & CGCG 122-067 & 0.089008 & 5 & 302 & MSPM 05544 & GClstr & 0.089190 & SPEC & 0.001 \\
\hline
\end{tabular}

\section{Notes.}

${ }^{\mathrm{a}}$ Number of galaxies within $1 \mathrm{Mpc}$ and $500 \mathrm{~km} \mathrm{~s}^{-1}$.

${ }^{\mathrm{b}}$ Number of galaxies within $10 \mathrm{Mpc}$ and $5000 \mathrm{~km} \mathrm{~s}^{-1}$.

${ }^{\mathrm{c}}$ Redshift type, from NED. EST—estimated, PHOT—photometric, and SPEC—-spectroscopic.

d The association of the super spiral galaxy with the compact group is based only on proximity on the sky. The group redshift in NED for SDSSCGB 59704 appears to be based only on the redshift of the super spiral. None of the other galaxies in SDSSCGB 16827 have measured redshifts. 


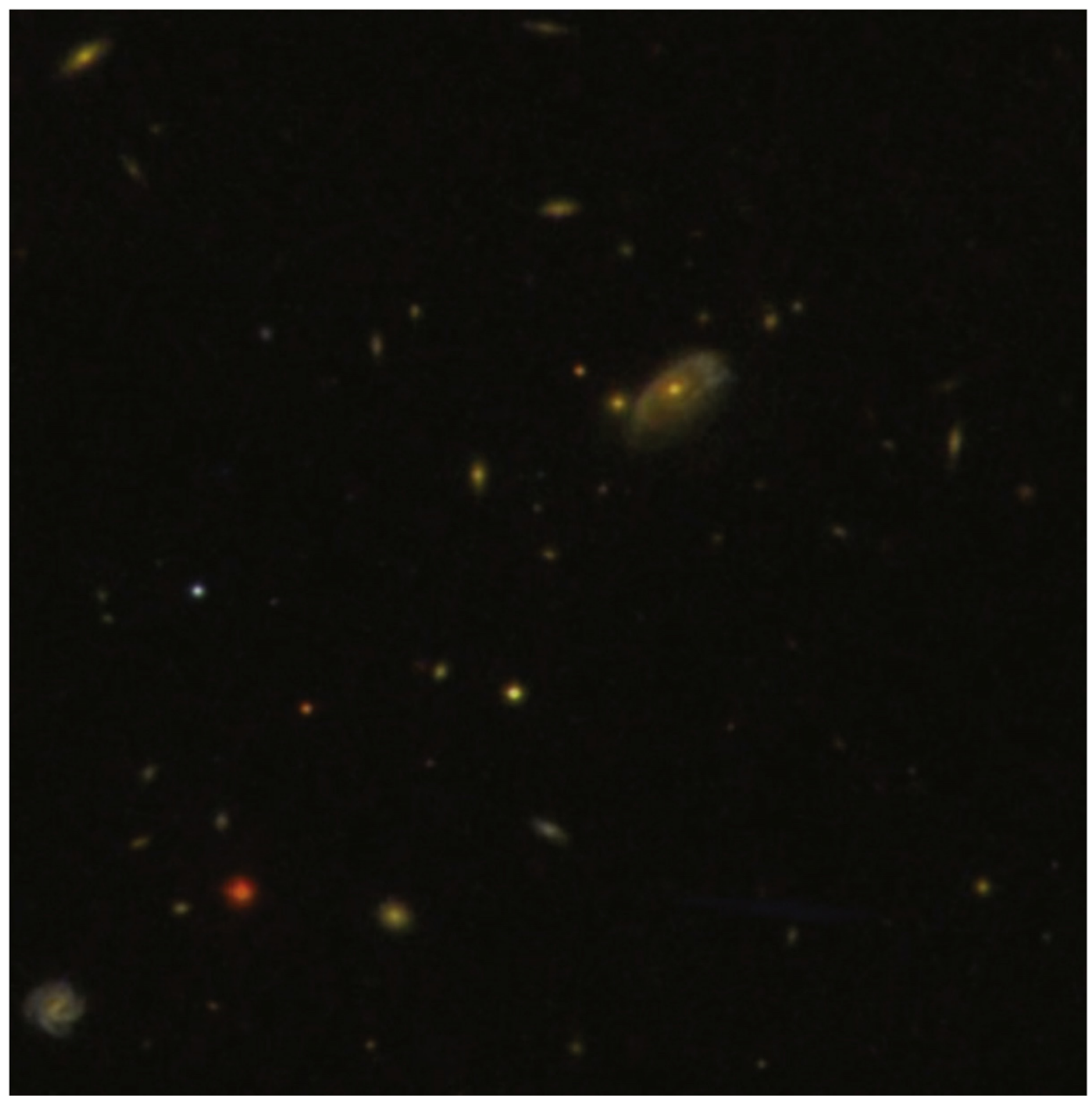

Figure 8. Super spiral 2MASX J11535621+4923562 (SS 17: $L_{r}=9.5 L^{*}, D=90 \mathrm{kpc}$ ) appears to be the brightest member of a previously unidentified galaxy cluster (OGC 0586 CLUSTER). Compare to the less-luminous cluster spiral galaxy SDSS J115407.96+492200.8 $\left(L_{r}=2.8 L^{*}, D=39 \mathrm{kpc}\right)$ in the lower left corner. The SDSS image is $203^{\prime \prime}(579 \mathrm{kpc})$ on each side.

galaxies and galaxy clusters within a sphere of comoving radius $10 \mathrm{Mpc}$. Because of the high redshifts of the super spirals, only the most luminous galaxies in their neighborhoods will tend to have measured spectroscopic redshifts in NED. We tabulate the number of galaxies (N1) with spectroscopic redshifts that are within $1 \mathrm{Mpc}$ and $500 \mathrm{~km} \mathrm{~s}^{-1}$, and the number (N10) within 10 projected Mpc and $5000 \mathrm{~km} \mathrm{~s}^{-1}$. The MSPM 05544 galaxy cluster, which appears to host the super spiral CGCG 122-067 (SS 50) has the largest number of cluster members with spectroscopic redshifts in NED (302), while the SDSSCGB 59704 galaxy group has the smallest number (2). These numbers should be taken as lower limits to the cluster membership, depending primarily on the SDSS spectroscopic selection limit and redshift.

There are likely more clusters to be discovered in the vicinity of super spirals. For example, a clear overdensity of galaxies is seen to the SE of 2MASX J11535621+4923562 (SS 17, Figure 8). We verify a concentration of 69 galaxies within
$10 \mathrm{Mpc}$ and $5000 \mathrm{~km} \mathrm{~s}^{-1}$ (Table 3: OGC 0586 CLUSTER), using NED's Environment tool. We estimate the mean redshift of OGC 0586 CLUSTER to be $z=0.166187$, from 12 galaxies with spectroscopic redshifts that are within 5 projected Mpc of SS 17.

\section{DISCUSSION}

While super spirals have similar structure to less luminous spiral galaxies, they are impressive in the vastness of their scale. A sense of how truly enormous these galaxies are can be gained by comparison to other galaxies in the same cluster (Figure 8: OGC 0586 CLUSTER). The 2MASX J11535621 +4923562 (SS 17) super spiral at $z=0.16673$, with a luminosity of $L_{r}=9.5 L^{*}$ and a diameter of $90 \mathrm{kpc}$, can be compared to a more common, less luminous spiral galaxy which has $L_{r}=2.8 L^{*}$ and a diameter of $39 \mathrm{kpc}$ (13!! 7), at about the same redshift $(z=0.16721)$. 


\subsection{Analogs}

It is natural to ask whether any analogs to super spirals have been found at lower redshift. One well-known example of a giant spiral galaxy is Malin $1(z=0.083)$, initially suggested to be a proto-disk galaxy because of its massive $\mathrm{H}$ i disk (Bothun et al. 1987). While Malin 1 does have one of the largest stellar disks known, with an exponential scale length of $70 \mathrm{kpc}$, its global $r$-band luminosity $\left(L_{r}=1.8 L^{*}\right)$ is not nearly great enough to make it into the OGC catalog. Its disk has very low surface brightness and is not readily visible in SDSS images. As further points of comparison, we estimate a global stellar mass of $1.2 \times 10^{10} M_{\odot}$ and global SFR of $1.2 M_{\odot} \mathrm{yr}^{-1}$, which are both much lower than the range spanned by super spirals.

Other giant spiral galaxies are found in the local universe, though they also have considerably lower luminosities than the super spirals in our sample. Romanishin (1983) find 107 spiral galaxies in the Uppsala General Catalog of Galaxies (UGC) at $z<0.05$, with cosmology-corrected $B$-band isophotal diameters (at $25.0 \mathrm{mag} \operatorname{arcsec}^{-2}$ ) of $D=65-150 \mathrm{kpc}$, similar to super spirals. The 39 giant UGC spirals with SDSS photometry in NED have $r$-band luminosities of $0.2-4.6 L^{*}$, stellar masses of $6 \times 10^{8}$ to $4 \times 10^{10} M_{\odot} \mathrm{yr}^{-1}$, and SFRs of 0.2-7.7 $M_{\odot} \mathrm{yr}^{-1}$. Because of their considerably lower stellar masses, they cannot be faded super spirals, but could be useful analogs for understanding giant disks. One of the largest giant spiral galaxies, UGC 2885 has a rotational velocity of $280 \mathrm{~km} \mathrm{~s}^{-1}$ at a radius of $60 \mathrm{kpc}$, and has undergone fewer than 10 rotations at its outer edge in the age of the universe (Rubin et al. 1980).

Super spirals may also be related to the cold sub-millimeter galaxies (SMGs) discovered at redshift $z=0.4-1$ (Chapman et al. 2002). The relatively cold $(\sim 30 \mathrm{~K})$ dust temperatures of these SMGs may indicate starburst activity in a disk rather than a spheroid. In comparison, the FIR SED of super spiral 2MASX J13275756+3345291 (SS 05, see the Appendix) is fit by the sum of a cold dust component with $T=21_{-1.8}^{+0.9} \mathrm{~K}$, likely from the disk, and a warmer dust component with temperature $T=50_{-2.8}^{+5.0} \mathrm{~K}$, likely from the starbursting bulge. GN20, one of the most luminous sub-millimeter detected star bursting galaxies, shows molecular gas and star formation distributed in a $10 \mathrm{kpc}$ scale disk at $z=4.05$ (Carilli et al. 2010). Deep near to mid-IR imaging of SMGs at intermediate redshifts will be necessary to measure their sizes and stellar masses and better determine their relationship to super spirals.

\subsection{Formation and Survival}

We estimate an average super spiral number density of $\sim 60$ $\mathrm{Gpc}^{-3}$ at $z<0.3$, correcting for $\sim 45 \%$ incompleteness at high disk inclination (Section 4.5). The space density of super spirals is therefore only $\sim 6 \%$ of the space density of elliptical galaxies in the same $r$-band luminosity range. Even the largest galaxy evolution simulations to date, such as the Illustris simulation (Vogelsberger et al. 2014; Snyder et al. 2015), covering $\sim 1.0 \times 10^{-3} \mathrm{Gpc}^{3}$, are not big enough to manufacture a significant number of super spirals. Therefore, no adequate prediction exists for the expected number of super spirals at $z<0.3$, nor are there simulations showing how these giant disk galaxies might form.

Super spirals could be formed by gas-rich major spiral-spiral mergers. Simulations that collide two gas-rich disk galaxies are able to produce post-merger spiral galaxies, albeit at smaller scale (Barnes 2002; Springel \& Hernquist 2005; Robertson et al. 2006; Hopkins et al. 2009). While merging stellar disks are typically destroyed, the gas in the outer disks may combine to reform an even larger gas and stellar disk. Orbital geometry may also be important, with misaligned or retrograde orbits leading to more gas-rich final merger products. If the dynamical timescales are longer and the merger-induced torques are even weaker in the outer disks of super spiral mergers, this may also be conducive to the preservation of gas disks and reformation of stellar disks. Alternatively, super spirals might be formed more gradually, from the inside out by accretion of cold gas. This may require a relatively low halo mass in order to avoid accretion shocks, which might prevent the gas from settling onto the outer disk Dekel \& Birnboim (2006). It will be important to study the spatial distribution of both neutral gas and star formation in super spirals to gain further insight into how their disks are formed.

It appears that the super spirals in our sample have so far avoided the fate of the vast majority of the most massive galaxies and continue to form stars in spite of their extreme mass, bucking the trend of cosmic downsizing. There are several possible reasons for this success. First, super spirals may be robust to mergers because of their massive, dissipative gaseous disks. It appears that several super spirals in our sample have survived recent major mergers with their starforming disks intact. Second, the supermassive black holes in super spiral bulges may not be large enough to provide enough feedback to drive away the gas in the giant galaxy disk. Third, the halo mass may not be large enough to cut off cold accretion onto the disk via accretion shocks. Finally, a large enough gas reservoir may have already settled into the disk to fuel star formation for a long time into the future. Observations across the electromagnetic spectrum are called for to distinguish among these possibilities.

\subsection{Connection to Quenched Disk Galaxies}

Super spirals occupy a relatively empty corner of the SFR versus stellar mass diagram (Figure 1(b)). They lie above an extrapolation of the star-forming main sequence, at the most extreme mass and SFR. We find that most super spirals have SSFR $>0.08 \mathrm{Gyr}^{-1}$. They are forming stars at a rate that would allow them to build up their mass in less than the age of the universe. This is unlike similarly massive, yet much more common disk galaxies (early type spirals and lenticulars) that fall below the star-forming main sequence, in what we shall call the disk quenching sequence (DQS: the disk galaxy subset of the green valley population). The disk-quenching sequence is discussed in the context of SSFR and UV color evolution by Schawinski et al. (2014), and in the context of IR color evolution by Alatalo et al. (2014). Quenching disk galaxies are likely greatly reduced in their ability to form stars because their supply of cold gas has been cut off (e.g., Dekel \& Birnboim 2006).

The most densely populated ridge of the DQS is close to the median stellar mass of our super spiral sample $\left(M_{\text {stars }}=1.1 \times 10^{11} M_{\odot}\right)$. We suggest that the majority of disk galaxies along this ridge were once super spirals. At a minimum, galaxies of this mass must have attained an average SFR $>7 M_{\odot} \mathrm{yr}^{-1}$ in order to have formed in less than the age of the universe. This would put them squarely in the SFR and SSFR range of super spirals. A further implication is that their $D=60-130 \mathrm{kpc}$ diameter stellar disks must have faded dramatically. If fossil giant disks are detected around massive 
lenticular galaxies with deep imaging, it will provide strong evidence for this hypothesis. In addition, deep $\mathrm{H}$ I and $\mathrm{CO}$ observations may reveal if their cold gas reservoir has been entirely depleted or reduced to a level that is not conducive to star formation.

\section{CONCLUSIONS}

We report the discovery of a large sample of the most optically luminous $\left(L_{r}>8 L^{*}\right)$, biggest, and most massive spiral galaxies in the universe, which we call super spirals. These galaxies are very rare $\left(\sim 60 \mathrm{Gpc}^{-3}\right)$, but are easily observed out to $z=0.3$ because of their high luminosities and gigantic sizes. Super spirals are forming stars at 5-65 $M_{\odot} \mathrm{yr}^{-1}$, a rate greater than their mean SFR over the age of the universe. Bulge-disk decompositions confirm the presence of giant stellar disks, with a median exponential scale length of $12.2 \mathrm{kpc}, 2.3$ times the median scale length of less luminous spirals at the same redshift. The bulge-to-total optical luminosity distribution is also significantly different for super spirals, showing a deficit of galaxies with $B / T<0.1$, and a concentration of galaxies with $B / T=0.1-0.2$. Roughly $11 \%$ of super spirals have Seyfert or QSO nuclei, suggesting that they are still actively adding mass to their supermassive black holes. We find evidence that several super spirals are undergoing major mergers, but manage to keep their star-forming disks intact, and avoid being transformed in to red-and-dead elliptical galaxies. Some super spirals are brightest cluster galaxies, while others appear to be isolated in the field. We suggest that super spirals may be the progenitors of red and dead lenticular galaxies of similar mass.

This work was made possible by the NASA/IPAC Extragalactic Database and the NASA/ IPAC Infrared Science Archive, which are both operated by the Jet Propulsion Laboratory, California Institute of Technology, under contract with the National Aeronautics and Space Administration. We thank Joe Mazzarella, Ben Chan, Marion Schmitz, and the rest of the NED team for useful discussions and their support of this work. This publication makes use of data from the Galaxy Evolution Explorer, retrieved from the Mikulski Archive for Space Telescopes (MAST). STScI is operated by the Association of Universities for Research in Astronomy, Inc., under NASA contract NAS5-26555. Support for MAST for non-HST data is provided by the NASA Office of Space Science via grant NNX09AF08G and by other grants and contracts. Funding for the Sloan Digital Sky Survey IV has been provided by the Alfred P. Sloan Foundation, the U.S. Department of Energy Office of Science, and the Participating Institutions. SDSS-IV acknowledges support and resources from the Center for High-Performance Computing at the University of Utah. The SDSS web site is www.sdss.org. This publication makes use of data products from the Two Micron All Sky Survey, which is a joint project of the University of Massachusetts and the Infrared Processing and Analysis Center/California Institute of Technology, funded by the National Aeronautics and Space Administration and the National Science Foundation. This publication makes use of data products from the Wide-field Infrared Survey Explorer, which is a joint project of the University of California, Los Angeles, and the Jet Propulsion Laboratory/California Institute of Technology, funded by the National Aeronautics and Space Administration. This work is based in part on observations made with the Spitzer Space Telescope, which is operated by the Jet Propulsion Laboratory, California Institute of Technology under a contract with NASA. We also make use of data from Herschel, an ESA space observatory with science instruments provided by European-led Principal Investigator consortia and with important participation from NASA. We thank Katey Alatalo for providing the SDSS-WISE comparison data in Figure 1(a), which is adapted from Alatalo et al. (2014). We thank Phil Hopkins and Ski Antonucci for insightful discussions that contributed to the manuscript. Finally, we thank the anonymous referee for suggesting that we analyze available bulge-disk decompositions by Simard et al. (2009), strengthening our results.

\section{APPENDIX CUSTOM PHOTOMETRY AND VALIDATION OF $M_{\text {stars }}$ AND SFR}

In order to validate our stellar mass and SFR estimates, which are based on $K \mathrm{~s}, u, r$, and WISE $12 \mu \mathrm{m}$ photometry, we make a more detailed analysis of two representative examples from our super spiral sample. We remeasure their photometry in matched apertures, rather than relying on catalog photometry. Then we fit their SEDs to make full use of the available multi-band photometry to estimate more accurate $M_{\text {stars }}$ and SFR. We chose SDSS J094700.08+254045.7 (SS 16) for this analysis because it is one of the brightest super spirals in our sample, with good photometry in many bands, and typical colors. The SDSS spectrum of its bulge is also typical of most super spirals, being dominated by an old stellar population (Figure 9). We also make a detailed study of 2MASX $\mathrm{J} 13275756+3345291$ (SS 05), which is the most luminous (non-QSO) mid-IR source in our sample and has an SDSS nuclear spectrum with strong young stellar component and high-equivalent width $\mathrm{H} \alpha$ emission (Figure 10), characteristic of starburst activity.

We remeasured GALEX (FUV, NUV), SDSS $(u, g, r, i, z)$, 2MASS $(J, H, K s)$ and WISE band 1-4 photometry for SS 16 using the SAOImager ds9 (Joye \& Mandel 2003) on images retrieved from MAST, SDSS, and IRSA (Figure 11). Aperture and color corrections were applied as necessary and the GALEX and SDSS photometry was corrected for foreground extinction due to the Milky Way dust (Stoughton et al. 2002; Wyder et al. 2005). The Galactic extinction is a modest $A_{V}=0.063$ mag (NED). We used an elliptical aperture with semimajor and semiminor axes of 31 ". 5 and 25 !. 5 , respectively, in order to capture the full flux of the spiral disk in all bands. This corresponds to major and minor diameters of $125 \mathrm{kpc}$ and $102 \mathrm{kpc}$. We also compute $3 \sigma$ IRAS upper limits based on the rms uncertainty measured by IRSA's Scan Processing and Integration tool (SCANPI) to constrain the FIR luminosity.

We present the SED of SS 16 in Figure 12. The galaxy is detected in all GALEX, SDSS, 2MASS, and WISE bands, but is undetected by IRAS. The UV through near-IR data points reveal a massive old stellar population plus a young stellar population. Mid-IR emission appears to be dominated by PAHs and warm dust from star formation. We fit the SED using MAGPHYS template fitting (da Cunha et al. 2008). This gives a total stellar mass of $1.8_{-0.2}^{+0.3} \times 10^{11} M_{\odot}$ and a SFR of $9.9_{-0.3}^{+1.6} M_{\odot} \mathrm{yr}^{-1}$. We get a consistent estimate of $1.2 \pm 0.1 \times 10^{11} M_{\odot}$ for the stellar mass from the $u-r$ color and $K s$ band luminosity, applying the Bell et al. (2003) prescription for color-dependent mass-to-light ratio 


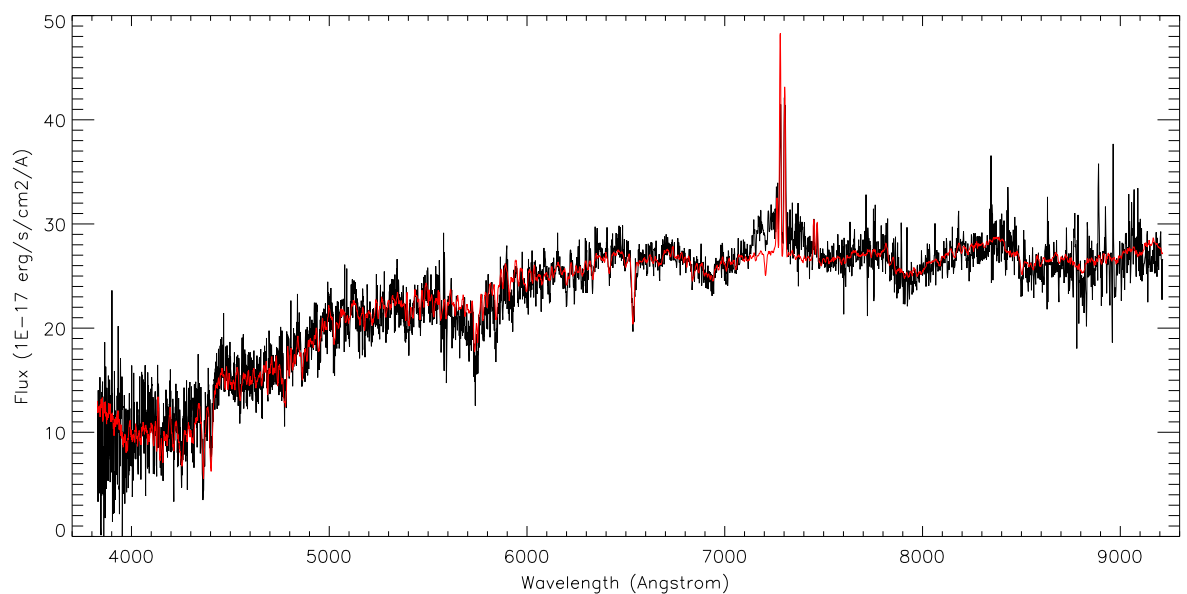

Figure 9. SDSS DR9 optical spectrum and spectral model of SDSS J094700.08+254045.7 (SS 16). Note the possible broad H $\alpha$ emission line not fit by the spectral model, indicative of a Seyfert 1 AGN.

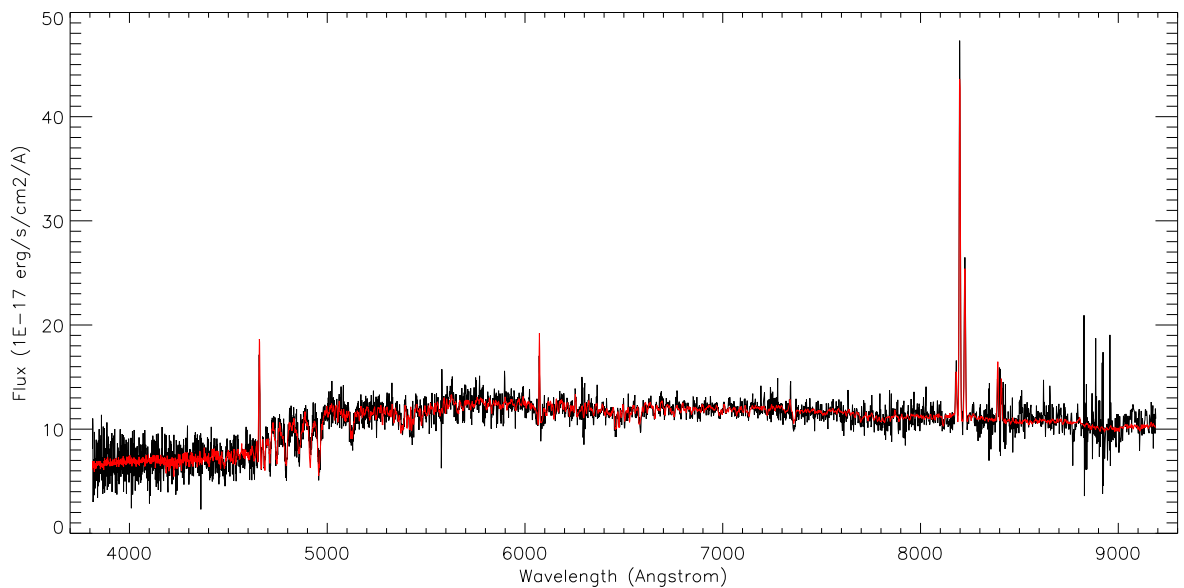

Figure 10. SDSS DR9 optical spectrum and spectral model of 2MASX J13275756+334529 (SS 05). The blue spectral slope and strong H $\alpha$ emission indicate starburst activity in the galaxy bulge that contributes to the high star formation rate in the galaxy as a whole.
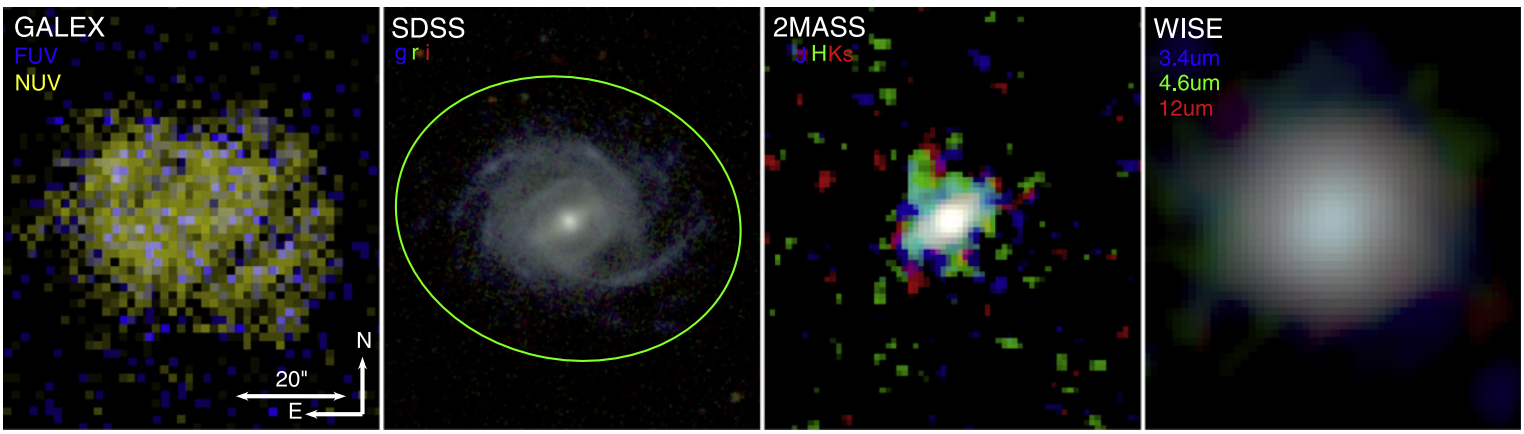

Figure 11. GALEX, SDSS, 2MASS and WISE images of SDSS J094700.08+254045.7 (SS 16). The image scale is $1.991 \mathrm{kpc} /{ }^{\prime \prime}$. The photometric aperture with major and minor axes of 125 and $102 \mathrm{kpc}$, respectively, is indicated by the ellipse on the SDSS image.

(Table 1). The WISE band 3 luminosity gives a consistent SFR of $13.5 \pm 0.2 M_{\odot} \mathrm{yr}^{-1}$, using the prescription of Chang et al. (2015). Lacking FIR detections, we do not have a good handle on the total dust mass, however, the SED fit formally yields a dust mass of $\sim 10^{8} M_{\odot}$, based on the PAH emission and FIR upper limits. This corresponds to roughly $\sim 10^{10} M_{\odot}$ of gas, assuming a standard gas/dust ratio of 100 .

We remeasured GALEX (FUV, NUV), SDSS ( $u, g, r, i, z)$, 2MASS $(J, H, K s)$ and WISE band 1-4 photometry for SS 05 (Figure 13), using a similar procedure. We also retrieved Spitzer IRAC and MIPS, and Herschel PACS and SPIRE images from the respective IRSA and ESA archives to measure the IR fluxes. We used a circular aperture with 20 ". 0 (156 kpc) radius for most bands. However, at SPIRE wavelengths, we used the larger point source apertures of $22^{\prime \prime}, 30^{\prime \prime}$, and $42^{\prime \prime}$, in order to contain the broader point-spread function. The Galactic extinction is only $A_{V}=0.034 \mathrm{mag}$ (NED).

We present the SED of SS 05 in Figure 14. The galaxy is detected in all measured bands except the SPIRE $500 \mu \mathrm{m}$ band. In contrast to SS 16, there is a stronger component of emission from young stars, and much more luminous IR emission from star formation activity. We fit the SED using MAGPHYS, yielding 


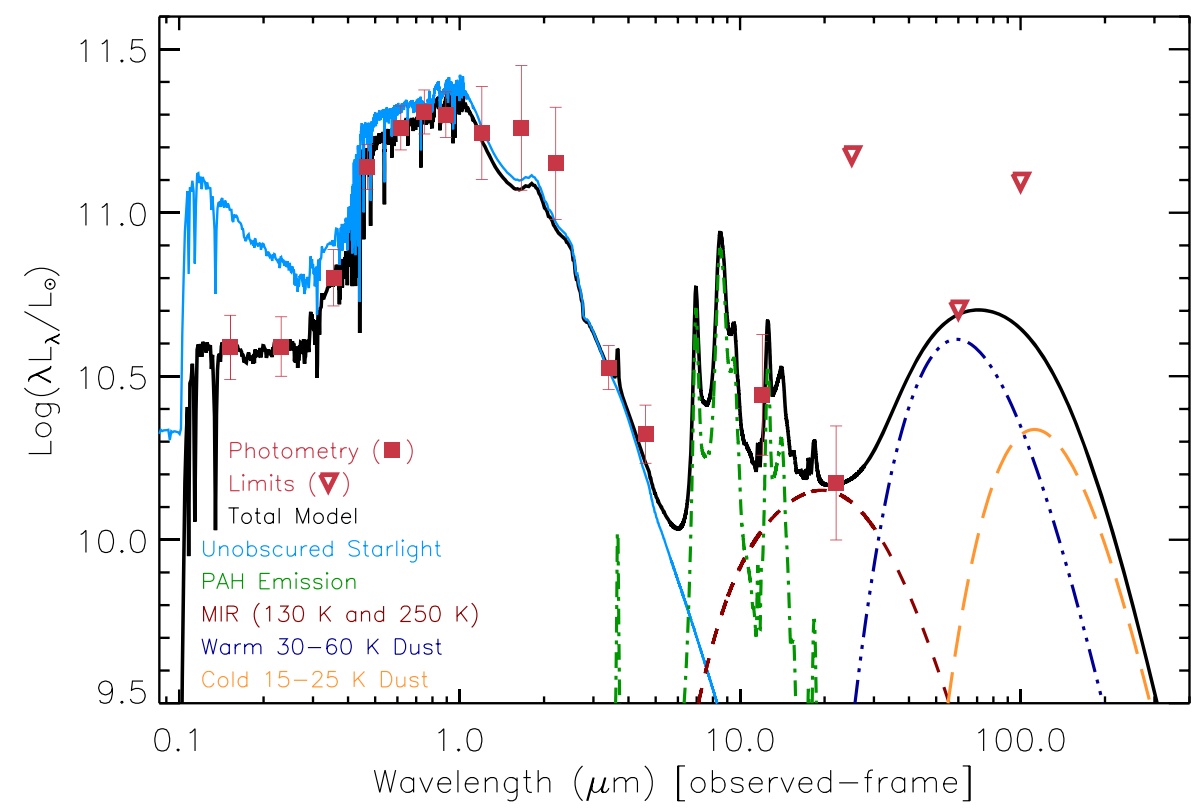

Figure 12. Spectral Energy Distribution of SDSS J094700.08+254045.7 (SS 16) fit by MAGPHYS. GALEX (NUV, FUV), SDSS ( $u, g, r, i, z), 2 \mathrm{MASS}(J, H, K \mathrm{~s})$, and WISE band 1-4 photometry are measured in the aperture shown in 11. IRAS upper limits at 25, 60, and $100 \mu \mathrm{m}$ are estimated using SCANPI.
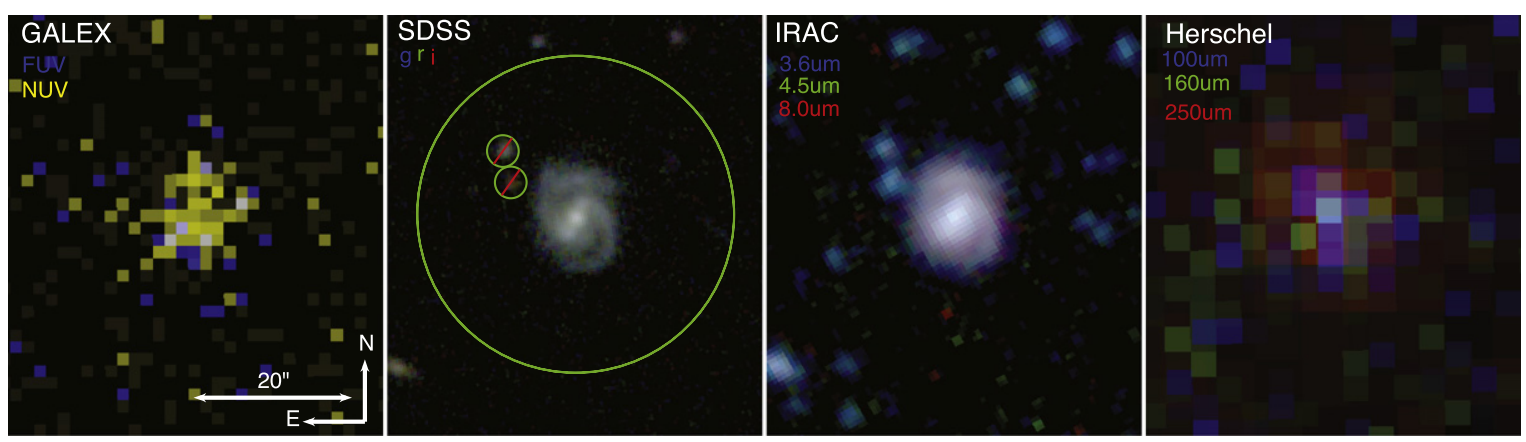

Figure 13. GALEX, SDSS, Spitzer IRAC, and Herschel images of 2MASX J13275756+334529 (SS 05). The image scale is 3.898 kpc/". The circular photometric aperture with diameter $156 \mathrm{kpc}$ and two exclusion regions are indicated on the SDSS image.

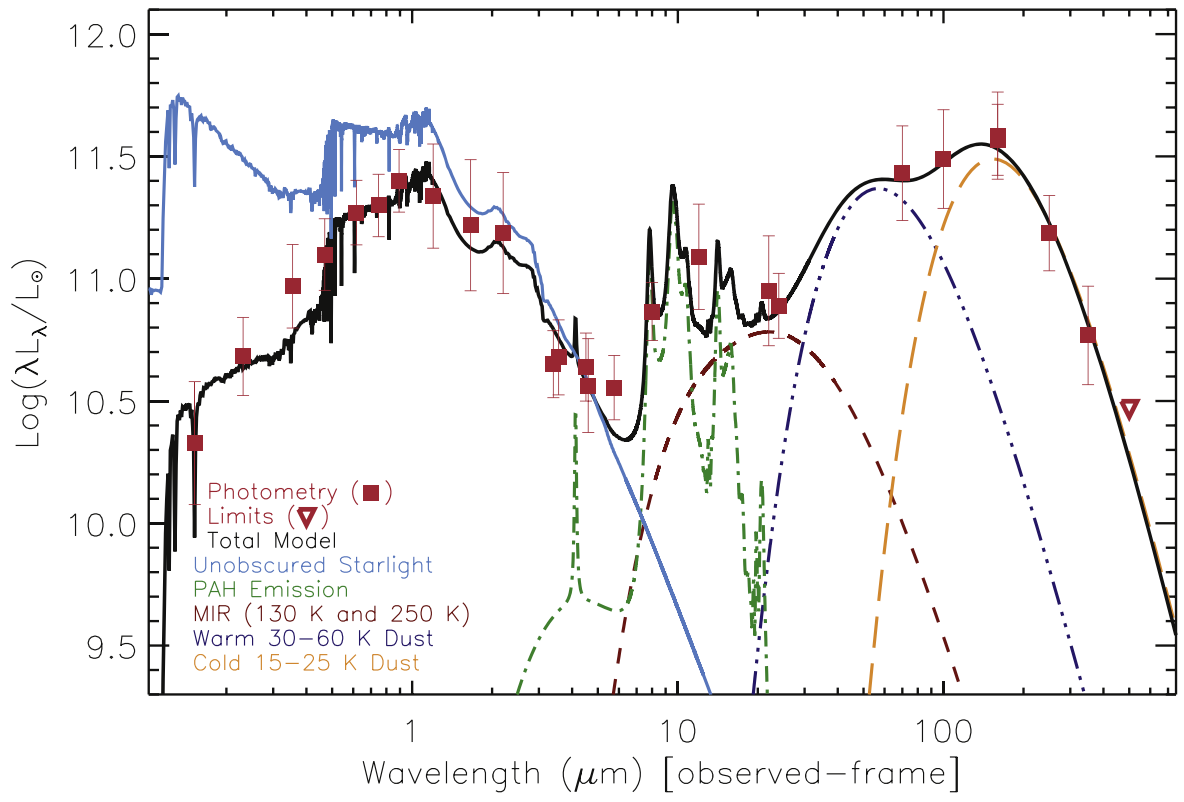

Figure 14. Spectral Energy Distribution of 2MASX J13275756+334529 (SS 05) fit by MAGPHYs. GALEX (NUV, FUV), SDSS ( $u, g, r, i, z), 2 \mathrm{MASS}(J, H, K \mathrm{~s})$, Spitzer IRAC and MIPS 24, 70, and $160 \mu \mathrm{m}$, WISE band 1-4, Herschel PACS 100 and $160 \mu \mathrm{m}$, and SPIRE photometry are measured in the aperture shown in Figure 13. SPIRE $500 \mu \mathrm{m}$ luminosity is an upper limit. 
a total stellar mass of $2.04_{-0.09}^{+0.05} \times 10^{11} M_{\odot}$ and SFR of $40.5_{-0.5}^{+6.5} M_{\odot} \mathrm{yr}^{-1}$. The stellar mass is consistent with the value of $1.6 \pm 0.3 \times 10^{11} M_{\odot}$ that we obtain from the $u-r$ color and $K s$ band luminosity (Table 1). The WISE [12] luminosity gives a somewhat larger SFR of $65 \pm 4 M_{\odot} \mathrm{yr}^{-1}$, using the conversion factor of Chang et al. (2015). The Herschel FIR measurements yield a secure estimate of total dust mass from the SED fit of $7_{-1}^{+3} \times 10^{8} M_{\odot}$, corresponding to $7 \times 10^{10} M_{\odot}$ of gas, assuming a standard gas/dust ratio of 100 .

\section{REFERENCES}

Alatalo, K., Cales, S. L., Appleton, P. N., et al. 2014, ApJL, 794, 13 Baldry, I. K., Glazebrook, K., Brinkmann, J., et al. 2004, ApJ, 600, 681 Barnes, J. 2002, MNRAS, 333, 481

Barnes, J. E., \& Hernquist, L. E. 1991, ApJL, 370, L65

Bell, E. F., McIntosh, D. H., Katz, N., \& Weinberg, M. D. 2003, ApJS, 149,289

Blanton, M. R., Hogg, D. W., Bahcall, N. A., et al. 2003, ApJ, 592, 819

Bothun, G. D., Impey, C. D., Malin, D. F., \& Mould, J. R. 1987, AJ, 94, 23

Brinchmann, J., Charlot, S., White, S. D. M., et al. 2004, MNRAS, 351, 1151

Carilli, C. L., Daddi, E., Riechers, D., et al. 2010, ApJ, 714, 1407

Chang, Y.-Y., van der Wel, A., da Cunha, E., \& Rix, H.-W. 2015, ApJS, 219, 8

Chapman, S. C., Smail, I., Ivison, R. J., et al. 2002, ApJ, 573, 66

da Cunha, E., Charlot, S., \& Elbaz, D. 2008, MNRAS, 339, 1595

Dekel, K., \& Birnboim, Y. 2006, MNRAS, 368, 2

Elbaz, D., Daddi, E., Le Borgne, D., et al. 2007, A\&A, 468, 33
Hopkins, P. F., Cox, T. J., Younger, J. D., \& Hernquist, L. 2009, ApJ, 691, 1168

Hopkins, P. F., Hernquist, L., Cox, T. J., et al. 2006, ApJS, 163, 1

Joye, W. A., \& Mandel, E. 2003, in ASP Conf. Ser. 295, Astronomical Data Analysis Software and Systems XII, ed. H. E. Payne, R. I. Jedrezejewsk, \& R. N. Hook (San Francisco, CA: ASP), 489

Lilly, S. J., Carollo, C. M., Pipino, A., Renzini, A., \& Peng, Y. 2013, ApJ, 772, 119

Lintott, C. J., Schawinski, K., Slosar, A., et al. 2008, MNRAS, 389, 1179

Ogle, P., Lanz, L., \& Appleton, P. 2014, ApJL, 788, L33

Ogle, P. M., Mazzarella, J., Ebert, R., et al. 2015, in ASP Conf. Ser. 495, Astronomical Data Analysis Software and Systems XIIV, ed. A. R. Taylor, \& E. Rosolowsky (San Francisco, CA: ASP), 25

Robertson, B., Bullock, J. S., Cox, T. J., et al. 2006, ApJ, 645, 986 Romanishin, W. 1983, MNRAS, 204, 909

Rubin, V. C., Ford, W. K., \& Thonnard, N. 1980, ApJ, 238, 471

Schawinski, K., Urry, C. M., Simmons, B. D., et al. 2014, MNRAS, 440, 889 Schechter, P. 1976, ApJ, 203, 297

Simard, L., Clowe, D., Desai, V., et al. 2009, A\&A, 508, 1141

Simard, L., Mendel, J. T., Patton, D. R., Ellison, S. L., \& McConnachie, A. W. 2011, ApJS, 196, 11

Sivanandam, S., Rieke, M. J., \& Rieke, G. H. 2014, ApJ, 796, 89

Snyder, G. F., Torrey, P., Lotz, J. M., et al. 2015, MNRAS, 454, 1886

Springel, V., \& Hernquist, L. 2005, ApJL, 622, L9

Stoughton, C., Lupton, R. H., Bernardi, M., et al. 2002, AJ, 123, 485

Strateva, I., Ivezic, Z., Knapp, G. R., et al. 2001, AJ, 122, 1861

Strauss, M. A., Weinberg, D. H., Lupton, R. H., et al. 2002, AJ, 124, 1810

Toomre, A., \& Toomre, J. 1972, ApJ, 178, 623

Vogelsberger, M., Genel, S., Springel, V., et al. 2014, MNRAS, 444, 1518

Wuyts, S., Forster Schreiber, N. M., van der Wel, A., et al. 2011, ApJ, 742, 96

Wyder, T. K., Treyer, M. A., Milliard, B., et al. 2005, ApJL, 619, L15 